\title{
Asymptotics for Optimal Design Problems for the Schrödinger Equation with a Potential
}

\author{
Alden Waters $\mathbb{D}^{1}$ and Ekaterina Merkurjev ${ }^{2}$ \\ ${ }^{1}$ Department of Mathematics, University of Groningen, Groningen, Netherlands \\ ${ }^{2}$ Department of Mathematics and CMSE, Michigan State University, East Lansing, MI, USA \\ Correspondence should be addressed to Alden Waters; alden.waters@gmail.com
}

Received 31 May 2018; Revised 3 September 2018; Accepted 8 October 2018; Published 18 October 2018

Academic Editor: Liwei Zhang

Copyright (c) 2018 Alden Waters and Ekaterina Merkurjev. This is an open access article distributed under the Creative Commons Attribution License, which permits unrestricted use, distribution, and reproduction in any medium, provided the original work is properly cited.

\begin{abstract}
We study the problem of optimal observability and prove time asymptotic observability estimates for the Schrödinger equation with a potential in $L^{\infty}(\Omega)$, with $\Omega \subset \mathbb{R}^{d}$, using spectral theory. An elegant way to model the problem using a time asymptotic observability constant is presented. For certain small potentials, we demonstrate the existence of a nonzero asymptotic observability constant under given conditions and describe its explicit properties and optimal values. Moreover, we give a precise description of numerical models to analyze the properties of important examples of potentials wells, including that of the modified harmonic oscillator.
\end{abstract}

\section{Introduction}

Let $\Omega \subset \mathbb{R}^{d}$ be a bounded domain with boundary $\partial \Omega$. Let $T>0$ and $\omega$ be a measureable subset of $\Omega$. We consider the Schrödinger equation with Dirichlet boundary conditions:

$$
\begin{aligned}
i \partial_{t} u & =\Delta u-V(x) u \\
u(0, x) & =u_{0}(x) \\
\left.u(t, x)\right|_{x \in \partial \Omega} & =0 .
\end{aligned}
$$

Here, $u: \mathbb{R} \times \Omega \longmapsto \mathbb{C}, V(x) \in L^{\infty}(\Omega)$, and $u_{0}(x)=u(0, x) \in$ $H_{0}^{1}(\Omega) \cap H^{2}(\Omega)$. In some instances, we will require higher regularity, but this will be specified when necessary.

Let $-\Delta$ indicate the Laplacian on the space $C_{0}^{\infty}(\Omega) \subset$ $L^{2}(\Omega)$. This operator is a symmetric operator acting on $L^{2}(\Omega)$ associated with the quadratic form

$$
\begin{aligned}
Q_{0}: H_{0}^{1}(\Omega) & \longrightarrow[0, \infty) \\
Q_{0}(f) & =\int_{\Omega} \nabla f(x) \cdot \overline{\nabla f(x)} d x .
\end{aligned}
$$

In particular we recall that the quadratic form is closable with respect to the norm

$$
Q_{D}(f)=\left(Q_{0}(f)+\|f\|_{L^{2}(\Omega)}\right)^{1 / 2} .
$$

The domain of the closure $Q_{D}(f)$ is the Sobolev space $H_{0}^{1}(\Omega)$. We can define the Dirichlet Laplacian $-\Delta_{D}$ via this extension procedure and moreover,

$$
\operatorname{Dom}\left(\left(-\Delta_{D}\right)^{1 / 2}\right)=\operatorname{Dom}(Q)=H_{0}^{1}(\Omega)
$$

If $\Omega$ is a bounded domain with boundary $\partial \Omega$ of class $C^{2}$ then

$$
\operatorname{Dom}\left(\left(-\Delta_{D}\right)\right)=H_{0}^{1}(\Omega) \cap H^{2}(\Omega) .
$$

All of the functions of this operator are interpreted via the Hilbert space functional calculus. In particular, $\exp \left(i t \Delta_{D}\right)$ is unitary, and we exploit this property to build our parametrices. The representation of the solutions presented here in the case of an added potential $V$ is new and relies on applications of advanced spectral theory.

If we consider the Schrödinger equation on a bounded domain $\Omega$ of $\mathbb{R}^{d}$ with Dirichlet boundary conditions, then 
observing the restriction of the solutions to a measurable subset $\omega$ of $\Omega$ during a time interval $[0, T]$ with $T>0$ is known as observability. Equation (1) is observable on $\omega$ in time $T$ if there exists $C>0$ such that

$$
C\left\|\partial_{t} u(0, x)\right\|_{L^{2}(\Omega)}^{2} \leq \int_{0}^{T} \int_{\omega}\left|\partial_{t} u(t, x)\right|^{2} d t d x .
$$

In previous literature, the above inequality is called the observability inequality when $V=0$.

It is well known that if the pair $(\omega, T)$ satisfies the observability inequality (6), then the energy of the solutions can be estimated in terms of the energy which is localized in $\omega \times(0, T)$. The search is then for the conditions on $\omega$ for which one can find the largest possible nonnegative constant for which inequality (6) holds.

We denote the observability constant by $C_{T}^{V}\left(\chi_{\omega}\right)$ to be the largest constant such that (6) holds. The constant can also be formulated as

$$
\begin{aligned}
& C_{T}^{V}\left(\chi_{\omega}\right)=\left\{\inf \frac{\int_{0}^{T} \int_{\omega}\left|\partial_{t} u(t, x)\right|^{2} d x d t}{\left\|\partial_{t} u(0, x)\right\|_{L^{2}(\Omega)}^{2}} \mid u(0, x)\right. \\
& \left.\quad \in H_{0}^{1} \cap H^{2}(\Omega)\right\} .
\end{aligned}
$$

The study of the observability constant $C_{T}^{V}\left(\chi_{\omega}\right)$ is important, since it gives an account for the well-posedness of the inverse problem of reconstructing $u$ from measurements over $[0, T] \times \omega$. In addition, we denote $C_{T}^{0}\left(\chi_{\omega}\right)$ as the constant associated with the Schrödinger equation without a potential. The main novelty of the paper is the analysis in the case of an added potential $V$.

We now connect the theory to a possible real-life application. Assume that $\Omega \subset \mathbb{R}^{d}$ is a cavity in which signals are propagating according to (1). To measure the propagating signals, one is allowed to place a few sensors into the cavity. We now assume that, in addition to the placement of the sensors, we are allowed to choose their shape. Therefore, the problem is now of determining the best possible location and shape of the sensors, which will obtain the best observation. Of course, the best choice is to observe the solutions over the whole domain $\Omega$. However, in practice, the domain scanned by the sensors is usually limited, for reasons such as the cost of such an operation. To make this limitation more mathematically precise, we consider measurable subsets of fixed size, i.e., subsets $\omega$ of $\Omega$ such that $|\omega|=L|\Omega|$, where $L \in(0,1)$. The subset $\omega$ represents the sensors in $\Omega$, and they are able to measure restrictions of the solutions of (1) to $\omega$.

Therefore, one and the most obvious way to model the problem of best observability is that of finding the optimal set which maximizes the functional $\chi_{\omega} \longrightarrow C_{T}^{V}\left(\chi_{\omega}\right)$ over the set

$$
\mathscr{M}_{L}=\{\omega \subset \Omega \mid
$$

$\omega$ is measurable and of Lebesgue measure $|\omega|$

$$
=L|\Omega|\} \text {. }
$$

However, we show that this problem not only is inherently difficult to solve, but is not so relevant in practice. Thus, we consider several modifications and simplifications of the model, to be described in the next section.

Optimal observation problems are found in numerous engineering applications, thus providing the motivation for our study. Examples include acoustics, piezoelectric actuators, vibration control in mechanical structures, damage detectors, and chemical reactions [1-5]. The goal is to optimize the type and place of the sensors in order to improve the estimation of the overall behavior of the state of the system.

The main contributions of the paper are the following:

(1) We present an elegant way to model the problem of best observability using the time asymptotic observability constant $C_{\infty}^{V}\left(\chi_{\omega}\right)$. We analyze the largest possible $C_{\infty}^{V}\left(\chi_{\omega}\right)$, over all $\omega \in \mathscr{M}_{L}$, and we develop conditions analogous to the quantum unique ergodicity conditions in [6] for this constant to hold.

(2) We demonstrate the conditions on the existence of a positive asymptotic observability constant $C_{\infty}^{V}\left(\chi_{\omega}\right)$ for an arbitrary subset $\omega$ of $\Omega$ and $T>0$, under certain requirements on the potential. Our results are supported by numerical experiments.

Remark 1. The paper [6] considers a variety of boundary conditions, but we focus on how to treat the problem with a potential, so we simply impose Dirichlet boundary conditions. Different boundary conditions will be the subject of future study. One could examine the problem on a compact Riemannian manifold $\mathscr{M}$, such that $(\mathscr{M}, g)$ has a boundary, and use the Laplace Beltrami-operator $\Delta_{g}$, and many of the same results would still hold. However, we let $\Omega$ be a subdomain of $\mathbb{R}^{d}$ for simplicity.

\section{Statement of the Main Theorems}

Consider the eigenvalues $\left(\lambda_{j}\right)_{j \in \mathbb{N}}$ and the corresponding eigenfunctions $\phi_{j}(x)$ for $-\Delta+V(x)$ on $\Omega$. Let $\left(\lambda_{j 0}\right)_{j \in \mathbb{N}}$ and $\phi_{j 0}(x)$ denote the eigenvalues and corresponding eigenfunctions of $-\Delta$ on $\Omega$. For the rest of this article we drop the subscript $D$ for the Dirichlet Laplacian.

We assume the $\phi_{j}(x)^{\prime} s$ are orthonormal and give references to classical spectral theory results which show that they can be used as a basis for $H_{0}^{1}(\Omega)$. The solution of (1) can then be represented as

$$
u(t, x)=\sum_{j=1}^{\infty} c_{j} \exp \left(i \lambda_{j} t\right) \phi_{j}(x)
$$

where $u(0, x) \in H_{0}^{1}(\Omega)$ is the initial data to the solution $u(t, x) \in C^{0}\left((0, T) ; H^{2}(\Omega)\right)$. The sequence $\left(c_{j}\right)_{j \in \mathbb{N}^{*}} \in \ell^{2}(\mathbb{C})$ is determined in terms of $u(0, x)$ as

$$
c_{j}=\int_{\Omega} u(0, x) \overline{\phi_{j}(x)} d x .
$$


Moreover,

$$
\left\|\partial_{t} u(0, x)\right\|_{L^{2}(\Omega)}^{2}=\sum_{j=1}^{\infty} \lambda_{j}^{2}\left|c_{j}\right|^{2}
$$

If

$$
G_{T}^{V}\left(\chi_{\omega}\right)=\int_{0}^{T} \int_{\omega}\left|\partial_{t} u(t, x)\right|^{2} d x d t
$$

then plugging in expansion (9) yields

$$
\begin{aligned}
G_{T}^{V}\left(\chi_{\omega}\right) & =\int_{0}^{T} \int_{\omega}\left|\sum_{j=1}^{\infty} \lambda_{j} c_{j} \exp \left(i \lambda_{j} t\right) \phi_{j}(x)\right|^{2} d x d t \\
& =\sum_{j, k=1}^{\infty} \lambda_{j} \lambda_{k} c_{j} \bar{c}_{k} \alpha_{j k} \int_{\omega} \phi_{j}(x) \overline{\phi_{k}(x)} d x
\end{aligned}
$$

with

$$
\begin{aligned}
\alpha_{j k} & =\int_{0}^{T} \exp \left(i\left(\lambda_{j}-\lambda_{k}\right) t\right) d t \\
& =\frac{2}{\lambda_{j}-\lambda_{k}}\left[\exp \left(i\left(\lambda_{j}-\lambda_{k}\right) T-1\right],\right.
\end{aligned}
$$

whenever $j \neq k$ and $\alpha_{j j}=T$ whenever $j=k$.

We notice that the determination of the observability constant is now a difficult spectral problem involving many inner products of eigenfunctions over the set $\omega$. Moreover, it is limited in practice, since the observability constant defined by (7) describes the worst possible case, which may not occur often in applications. In order to examine the problem further, one can consider the following simplifications:

(1) One can examine the problem of maximizing $G_{T}^{V}\left(\chi_{\omega}\right)$ over all possible measurable subsets $\omega \in \mathscr{M}_{L}$, given fixed initial data. In this case, if the optimal set exists, it depends on the initial data that is considered. This problem is still challenging, and also not relevant enough in practice, since initial data is not expected to be fixed, but uniform in nature. Therefore, we focus on the following second simplification, where all initial conditions are taken into account.

(2) One can instead consider a time asymptotic observability constant $C_{\infty}^{V}\left(\chi_{\omega}\right)$, as in [6]. The constant is defined as

$$
\begin{aligned}
& C_{\infty}^{V}\left(\chi_{\omega}\right)=\left\{\inf \lim _{T \rightarrow \infty} \frac{1}{T} \frac{\int_{0}^{T} \int_{\omega}\left|\partial_{t} u(t, x)\right|^{2} d x d t}{\left\|\partial_{t} u(0, x)\right\|_{L^{2}(\Omega)}^{2}} \mid\right. \\
& \left.u(0, x) \in H_{0}^{1} \cap H^{2}(\Omega)\right\} .
\end{aligned}
$$

This constant is the nonnegative constant for which the time asymptotic observability inequality

$$
\begin{aligned}
& C_{\infty}^{V}\left(\chi_{\omega}\right)\left\|\partial_{t} u(0, x)\right\|_{L^{2}(\Omega)}^{2} \\
& \quad \leq \lim _{T \rightarrow \infty} \frac{1}{T} \int_{0}^{T} \int_{\omega}\left|\partial_{t} u(t, x)\right|^{2} d x d t
\end{aligned}
$$

holds for every $u(0, x) \in H_{0}^{1}(\Omega) \cap H^{2}(\Omega)$. This is where we use the additional assumption $u \in H^{2}(\Omega)$ so the constant is well-defined. If $\bar{\Omega}$ is of class $C^{2}$ then this is the entire domain anyway. Shortly, we will show that the time asymptotic observability constant is equal to the randomized observability constant

$$
J^{V}\left(\chi_{\omega}\right)=\inf _{j \in \mathbb{N}} \int_{\omega} \phi_{j}^{2}(x) d x .
$$

Note that, from the definition of the observability constant, one obtains the following inequality:

$$
\lim \sup _{T \rightarrow \infty} \frac{C_{T}^{V}\left(\chi_{\omega}\right)}{T} \leq C_{\infty}^{V}\left(\chi_{\omega}\right) .
$$

The randomized observability constant can be derived in the following way. We introduce a field of i.i.d random variables $\left\{\beta_{j}\right\}_{j \in \mathbb{N}} \in\{0,1\}$ which we use to multiply the values of the initial data. Then

$$
\begin{aligned}
& \inf \lim _{T \rightarrow \infty} \frac{1}{T} \mathbb{E}\left(G_{T}^{V}\left(\chi_{\omega}\right)\right)=\inf \lim _{T \rightarrow \infty} \frac{1}{T} \mathbb{E} \\
& \cdot\left(\int_{0}^{T} \int_{\omega}\left|\sum_{j \in \mathbb{N}^{*}}(-\Delta+V)\left(c_{j} \beta_{j} \phi_{j}(x)\right)\right|^{2} d x d t\right)= \\
& \inf \lim _{T \rightarrow \infty} \frac{1}{T} \mathbb{E}\left(\int_{0}^{T} \int_{\omega}\left|\sum_{j \in \mathbb{N}^{*}} c_{j} \lambda_{j} \beta_{j} \phi_{j}(x)\right|^{2} d x d t\right)
\end{aligned}
$$

Note that (19), under the conditions

$$
\begin{aligned}
\left\{\beta_{j}\right\}_{j \in \mathbb{N}} & \in\{0,1\}, \\
\sum_{j}\left|c_{j} \lambda_{j}\right|^{2} & =1,
\end{aligned}
$$

is exactly $J^{V}\left(\chi_{\omega}\right)=\inf _{j \in \mathbb{N}} \int_{\omega} \phi_{j}^{2}(x) d x$.

We now state the main theorems of the paper. All of the theorems in this section are formulated for the Schrödinger equation with a potential, which is the main novelty. The first two results concern an expression for time asymptotic observability constant $C_{\infty}^{V}\left(\chi_{\omega}\right)$.

Theorem 2 (analogue to Theorem $2.6[6]$ ). For every measureable subset $\omega$ of $\Omega$,

$$
\begin{aligned}
& C_{\infty}^{V}\left(\chi_{\omega}\right) \\
& \quad=\left\{\inf \frac{\int_{\omega} \sum_{\lambda \in U}\left|\sum_{k \in I(\lambda)} c_{k} \phi_{k}(x)\right|^{2} d x}{\sum_{k=1}^{\infty}\left|c_{k}\right|^{2}} \mid\left(c_{j}\right)_{j \in \mathbb{N}^{*}}\right. \\
& \left.\quad \in \ell^{2}(\mathbb{C}) \backslash\{0\}\right\},
\end{aligned}
$$

where $U$ is the set of all distinct eigenvalues $\lambda_{k}$ and $I(\lambda)=\{j \in$ $\left.\mathbb{N}^{*} \mid \lambda_{j}=\lambda\right\}$. 
The proof of the theorem is in Section 4. If we set

$$
J^{V}\left(\chi_{\omega}\right)=\inf _{j \in \mathbb{N}} \int_{\omega} \phi_{j}^{2}(x) d x
$$

then similarly to Theorem 1 in [6], we have the following.

Corollary 3 (analogous to Corollary 2.7 in [6]). The inequality $C_{\infty}^{V}\left(\chi_{\omega}\right) \leq J^{V}\left(\chi_{\omega}\right)$ is true for every measurable subset $\omega$ of $\Omega$. If the domain $\Omega$ is such that every eigenvalue of $-\Delta+V$ is simple, then

$$
C_{\infty}^{V}\left(\chi_{\omega}\right)=\inf _{j \in \mathbb{N}} \int_{\omega} \phi_{j}^{2}(x) d x=J^{V}\left(\chi_{\omega}\right)
$$

for every measurable subset $\omega$ of $\Omega$. This shows that the offdiagonal terms in the eigenfunction expansion contribute less in the infinite time asymptotic regime.

The more difficult problem is using known results from perturbation theory to find a nonzero observability constant. We show that if $V(x)=\varepsilon V_{0}(x)$ for some $\varepsilon \in(0,1)$, then, under certain conditions on $\varepsilon$, we can find a positive time asymptotic observability constant for the Schrödinger equation whenever the corresponding operator without the potential $(V(x) \equiv 0)$ has one.

For Theorem 4 it is assumed that the potential has regularity $V \in L^{\infty}(\Omega)$. Let $C\left(V_{0}, \Omega\right)$ be a constant which depends uniformly on the diameter of $\Omega$ and the $L^{\infty}(\Omega)$ norm of $V_{0}$. This constant will be derived and given explicitly during the course of the proof. We prove the following.

Theorem 4. We assume that $-\Delta$ and $-\Delta+V(x)$ on $\Omega$ with Dirichlet boundary conditions both have simple spectra, for all $\varepsilon \in\left(0, \varepsilon_{0}\right)$ with fixed $\varepsilon_{0}$ sufficiently small. When $V(x)=$ $\varepsilon V_{0}(x)$, supp $V_{0} \subset \omega$, and $\varepsilon<1 / C\left(V_{0}, \Omega\right)$, the constant $J^{V}\left(\chi_{\omega}\right)$ is such that $J^{V}\left(\chi_{\omega}\right)>0$ if and only if $J^{0}\left(\chi_{\omega}\right)>0$ for the Schrödinger equation with $V(x) \equiv 0$.

The proof of Theorem 4 is in Section 7. Moreover, in Section 7, we discuss why the assumption that the spectra are simple is spectrally sharp, as there are counter-examples to the statement of Theorem 4 for nonsimple spectra given as a result of [7-9]. There is also an appendix on convergence of numerical algorithms using these functionals.

We also consider a relaxation of the problem. In particular, let $\overline{\mathscr{M}}_{L}$ be the convex closure of the set $\mathscr{M}_{L}$ in the $L^{\infty}$ weak star topology:

$$
\overline{\mathscr{M}}_{L}=\left\{a \in L^{\infty}(\Omega,[0,1])\left|\int_{\Omega} a(x) d x=L\right| \Omega \mid\right\} .
$$

We set

$$
\inf _{j \in \mathbb{N} *} \int_{\Omega} a(x) \phi_{j}^{2}(x) d x=J_{\varepsilon}(a)
$$

and also

$$
\inf _{j \in \mathbb{N} *} \int_{\Omega} a(x) \phi_{j 0}^{2}(x) d x=J(a) .
$$

We then have the following.
Theorem 5. Let $V=\varepsilon V_{0}(x)$ with $V_{0}(x) \in L^{\infty}(\Omega)$, with no assumption on the support of $V(x)$ in $\Omega$. We assume $-\Delta$ and $-\Delta+V(x)$ with Dirichlet boundary conditions both have simple spectrum, all $\varepsilon \in\left(0, \varepsilon_{0}\right)$ with fixed $\varepsilon_{0}$ sufficiently small. It follows that for any $a \in \bar{M}_{L}$

$$
\left|J_{\varepsilon}(a)-J(a)\right| \leq C_{1}\left(V_{0}, \Omega\right) \varepsilon^{2}
$$

with $C_{1}\left(V_{0}, \Omega\right)$ a constant depending only on $L^{\infty}(\Omega)$ norm of $V_{0}$ and $|\Omega|$. As a consequence, we can conclude

$$
\left|\max _{a \in \overline{\mathscr{M}}_{L}} J_{\varepsilon}(a)-\max _{a \in \overline{\mathscr{M}}_{L}} J(a)\right| \leq C_{1}\left(V_{0}, \Omega\right) \varepsilon^{2}
$$

The proof of Theorem 5 is in Section 7. Notice that we cannot show $J_{\varepsilon}(a)=0$ iff $J(a)$ is zero because we do not have such fine control over the $\mathcal{O}\left(\varepsilon^{2}\right)$ terms unless $a(x)=\chi_{\omega}(x)$.

In the last section, using existing software, we show explicit computations and an explicit representation of the observability constant for a variety of potentials including a damped harmonic oscillator. While the problem for the nonlinear Schrödinger equation has been investigated from the control theory standpoint [10-13], to the best of the authors knowledge, the problem of observability for potentials has not been addressed in an explicit way using eigenfunctions and numerical methods. Observability for the linear Schrödinger equation was examined in [14]. Our analysis extends their results in the linear case.

2.1. Comparison with Previous Literature. Let $\omega \subset \Omega$ be any nonempty open set and $T>0$; then there exists a constant $K_{T}\left(\chi_{\omega}\right)$ such that for any $u_{0} \in H_{0}^{1}(\Omega)$ we have

$$
\left\|u_{0}\right\|_{L^{2}(\Omega)}^{2} \leq K_{T}\left(\chi_{\omega}\right) \int_{0}^{T}\left\|\exp (i t \Delta) u_{0}\right\|_{L^{2}(\omega)}^{2} d t
$$

or a constant $B_{T}\left(\chi_{\omega}\right)$ such that for any $u_{0} \in H_{0}^{1}(\Omega) \cap H^{2}(\Omega)$

$$
\left\|\Delta u_{0}\right\|_{L^{2}(\Omega)}^{2} \leq B_{T}\left(\chi_{\omega}\right) \int_{0}^{T} \| \Delta\left(\exp (\text { it } \Delta) u_{0}\right) \|_{L^{2}(\omega)}^{2} d t
$$

depending on the domain of the operator.

In general the work of Lebeau [15] showed that control (the dual statement to the existence of positive constant $B_{T}\left(\chi_{\omega}\right)$ or $\left.K_{T}\left(\chi_{\omega}\right)\right)$ for the Schrödinger equation with or without the potential holds under the Geometric Control Condition (GCC):

(i) There exists $L=L(\Omega, \omega)>0$ such that every geodesic of length $L$ on $\Omega$ intersects $\omega$.

Therefore if we let $K_{T}^{V}\left(\chi_{\omega}\right)$ denote the constant with the potential, then $K_{T}^{V}\left(\chi_{\omega}\right)>0$ as soon as the GCC is satisfied. The GCC is also necessary in the case of a smooth potential when the geodesic flow is periodic [9]. For the flat torus, Jaffard [16] and Haraux [17] in 2D and Komoronik [18] in higher dimensions have shown that this not necessary: observability holds for any open set $\omega$. Their work was extended to operators with smooth potentials in $[19,20]$, and 
also for higher dimensions and time-dependent potentials in [21], and for irrational torii and general Schrördinger operators in [22]. One can see [23] for a literature review and extension to hyperbolic manifolds.

We look at the constant given by (7), which we are examining to be a different observability constant when the potential is present and this is distinct from that examined in previous literature. However it is closely related to context analyzed in [24] which is also done for time dependent potentials on the flat disk and other works. Therefore, the main goal here is to identify in which sense the randomized observability constant with the potential and that without are close.

When there is no potential, our formulation of the observability constant coincides with the definition (31). Indeed, for our formulation, one can rewrite (7) as

$$
\begin{aligned}
& C_{T}^{V}\left(\chi_{\omega}\right) \\
& \quad=\left\{\inf \frac{\int_{0}^{T} \int_{\omega}\left|(-\Delta+V) \exp (i t(-\Delta+V)) u_{0}\right|^{2} d x d t}{\left\|(-\Delta+V) u_{0}\right\|_{L^{2}(\Omega)}^{2}} \mid\right. \\
& \left.u_{0} \in H_{0}^{1} \cap H^{2}(\Omega)\right\}
\end{aligned}
$$

The positivity of this constant is not directly equivalent to the other two when $V$ is nonzero, as $V$ does not commute with $\exp (i t(-\Delta+V))$. The only time the existence of the constants $K_{T}^{V}\left(\chi_{\omega}\right)$ and $B_{T}^{V}\left(\chi_{\omega}\right)$ could imply the positivity of $C_{T}^{V}\left(\chi_{\omega}\right)$ directly is when the potential is positive. However, in the important aforementioned literature $[8,9,15,21,22,24]$, there are several cases in which conditions that ensure the positivity of these constants are equivalent-manifolds with periodic geodesic flow, flat tori, and the Euclidean disk. In all these cases, the geometric conditions on the observation set $\omega$ do not depend on the presence of the potential, regardless of whether or not this potential is positive or not, c.f. the introduction to [8].

Moreover, Theorem 4 is proved for the randomised observability constant $J^{V}\left(\chi_{\omega}\right)$ (otherwise known as the observability constant for eigenfunctions [7]). It is doubtful such a strong statement is true for the full observability constants $C_{T}^{V}\left(\chi_{\omega}\right)$ and $C_{T}^{0}\left(\chi_{\omega}\right)$ as the presence of cross terms in (14) is difficult to control when $\lambda_{j}^{\prime} s$ are large. Once again, as in [25], the randomized constant can be viewed as the optimistic best case scenario.

Since Theorem 4 is only true in the case of sufficiently small and regular potentials of compact support, this shows that even in the case of randomised initial data the observability constants (eigenfunction observability constants) can be very close for strong conditions relating $V$ and $\omega$. It is not that the eigenfunction constants cannot be close for $\operatorname{supp}(V)$ not contained in $\omega$; it is just that the current technique gives much less information about controlling the constants in terms of each other. Hence, Theorem 5 has a weaker formulation of the relationship of the relaxed constant $J_{\varepsilon}(a)$ with $\varepsilon$ dependent potential to the original one $J(a)$, and there is no assumption on the support of $V$ with respect to $\omega$. In general, showing observability for randomized initial data (otherwise known as observability of eigenfunctions) is possible under conditions on the observation region $\omega$ which are independent of $V$ for generic potentials, c.f. [8].

The main tools in this article are opposite those of the general tract of semiclassical analysis papers. Previous techniques take advantage of the spectral theorem to turn the high frequency eigenvalues $\lambda_{j}$ into the semiclassical parameter $h^{-2}$. Heuristically $-h^{2} \Delta+h^{2} V$ as a semiclassical operator has symbol $|\xi|^{2}+h^{2} V$, while $-h^{2} \Delta+\varepsilon h^{2} V$ has symbol $|\xi|^{2}+h^{2} \varepsilon V$ but in the latter case the Hamiltonian ray path $x(t)$ over which solutions are concentrated can be made sufficiently close to that of $|\xi|^{2}$ if $\varepsilon$ is sufficiently small, as long as $h \leq 1$ which is proved in [26], Lemma 8.3. The methodology in [26] fails here because approximate solutions can only be constructed under a nontrapping condition.

Because we are exploiting the small parameter $\varepsilon$, we use classical perturbation theory techniques rather than semiclassical analysis. Here we see that classical perturbation theory gives new information in the case when the eigenvalues are simple, which cannot be explained by entirely semiclassical techniques. Moreover the results are applicable to any eigenfunction/eigenvalue pair, not just the high frequency ones.

However, in this particular case examined in this article, if we rescale so that $h=\lambda^{-1 / 2}$, then the eigenvalue/eigenvector problem becomes $\left(-h^{2} \Delta+h^{2} V\right) u=u$, with symbol $|\xi|^{2}+$ $h^{2} V(x)$, which in the case of the two-dimensional flat disk, and the surface of a sphere, can be solved almost explicitly using semiclassical methods to a high degree of success, c.f. $[7,8]$, corresponding to high frequency eigenvalues in this scenario. In other geometries this is not the case, and these are the settings which we seek to begin to resolve in this article.

\section{Review of Spectral Theory}

Suppose $\Omega \subset \mathbb{R}^{d}$ is a bounded domain in $\mathbb{R}^{d}$. Then, as in the introduction the Laplace operator $-\Delta$ with Dirichlet boundary conditions can be defined as the self-adjoint operator with the quadratic form $Q_{0}(f)$

$$
Q_{0}(f)=\langle\nabla f, \overline{\nabla f}\rangle_{L^{2}(\Omega)}
$$

with domain $H_{0}^{1}(\Omega)$. Because the space $H_{0}^{1}(\Omega)$ is compactly embedded in $L^{2}(\Omega)$ by Rellich's theorem, the spectrum of this operator is purely discrete and has infinity as its only possible accumulation point, c.f. [27] for a review. Hence, there exists an orthonormal basis $\left(\phi_{j 0}\right)_{j \in \mathbb{N}}$ in $L^{2}(\Omega)$ consisting of eigenfunctions with eigenvalues $\left(\lambda_{j}\right)$, which we assume to be ordered:

$$
\begin{aligned}
-\Delta \phi_{j 0} & =\lambda_{j 0} \phi_{j 0} \\
\left\|\phi_{j 0}\right\|_{L^{2}(\Omega)} & =1 \\
\left.\phi_{j 0}\right|_{\partial \Omega} & =0 \\
0 & \leq \lambda_{10} \leq \lambda_{20} \leq \cdots
\end{aligned}
$$


Recall that a linear subspace $\mathscr{D}$ of the domain of a closed quadratic form $Q$ is called a core for $Q$ if $Q$ is the closure of its restriction to $\mathscr{D}$. We now recall the following result from [28].

Theorem 6 (Thm 8.2.1 in [28]). If $0 \leq V \in L_{\text {loc }}^{1}(\Omega)$ and $\Omega \subset$ $\mathbb{R}^{d}$ is a domain in $\mathbb{R}^{d}$, then the quadratic form

$$
\begin{gathered}
Q_{V}(f)=Q_{0}(f)+Q_{1}(f)= \\
\int_{\Omega}|\nabla f|^{2}+V|f|^{2} d x,
\end{gathered}
$$

which is defined on

$$
\operatorname{Dom}\left(Q_{V}\right)=\operatorname{Dom}\left(Q_{0}\right) \cap \operatorname{Dom}\left(Q_{1}\right),
$$

is the form of a nonnegative self-adjoint operator $H$. The space $C_{c}^{\infty}(\Omega)$ is a core for $Q$.

Remark 7. We could reduce the assumption on the potential from $L^{\infty}(\Omega)$ to $L_{l o c}^{1}(\Omega)$ using the above theorem in many of the following sections.

We also require the following useful result on self-adjoint operators from the same monograph [28].

Theorem 8 (Theorem 8.2.3, Corollary 4.4.3, [28]). If $H$ is defined on $L^{2}(\Omega)$ by $H f=-\Delta f+V f$, where $V \in L^{\infty}$, then $H$ is a self-adjoint and bounded below with the same domain as $H_{0}=-\Delta$.

We also have the following.

Theorem 9 (Thm 6.3.1 in [28]). For all bounded domains $\Omega \subset \mathbb{R}^{d}$, the operator $-\Delta$ has an empty essential spectrum and compact resolvent. The eigenvalues $\left\{\lambda_{n}\right\}_{n=1}^{\infty}$ of $-\Delta$ written in increasing order and repeated according to multiplicity satisfy

$$
b_{1} n^{2 / d} \leq \lambda_{n} \leq b_{2} n^{2 / d}
$$

for some $b_{1}, b_{2}>0$ depending only on the geometry of $\Omega$ and $n \geq 1$.

As such, $b_{1}$ and $b_{2}$ can be made arbitrarily close to one another, if $n$ is large, c.f. the proof of Theorem 6.3.1 in [28]. The eigenvalues of $-\Delta$ depend monotonically upon the region $\Omega$ and so can be bounded above and below by the eigenvalues of the cubes which are contained in (and, respectively, contain) $\Omega$. It follows from Theorem 8 that $H_{0}^{1}(\Omega)=\operatorname{Dom} Q(f)$. From this fact and the spectral theorem, we can conclude from Theorem 6 the following.

Corollary 10. For $V \in L^{\infty}(\Omega)$, if

$$
\lambda_{0} \leq \cdots \leq \lambda_{j} \leq \lambda_{j+1} \cdots
$$

with $\left\{\phi_{j}\right\}_{j \in \mathbb{N}}$ an orthonormal Hilbert basis of $H_{0}^{1}(\Omega)$ consisting of eigenfunctions of the Dirichlet operator $-\Delta+V$ on $\Omega$, which is associated with the eigenvalues $\left\{\lambda_{j}\right\}_{j \in \mathbb{N}}$, then we can write the propagated solution as

$$
u(t, x)=\sum_{j \in \mathbb{N}} c_{j} \phi_{j}(t, x)
$$

with

$$
c_{j}=\int_{\Omega} u(0, x) \overline{\phi_{j}(x)} d x .
$$

We use the basis properties in Corollary 10 in the next section.

\section{Proof of Theorem 2}

The basic idea is to use

$$
u(t, x)=\sum_{j=1}^{\infty} c_{j} \exp \left(i \lambda_{j} t\right) \phi_{j}(x)
$$

as the decomposition for the solution of (1), where $\left(\lambda_{j}, \phi_{j}(x)\right)_{j \in \mathbb{N}^{*}}$ are the eigenvalue and eigenfunction pairs for the $-\Delta+V$ operator. One can apply similar steps to [6] to prove Theorem 2 . Using a standard density argument, the approximation which holds over a finite number of modes,

$$
u(t, x) \approx \sum_{j=1}^{N} c_{j} \exp \left(i \lambda_{j} t\right) \phi_{j}(x)
$$

is enough to describe an observability constant which is valid in the large-time regime. Then, we use previously derived facts about perturbation theory to prove the other theorems.

Proof of Theorem 2. We start with the case when $-\Delta+V$ has simple eigenvalues. This proof is a simplification of the analogous theorem in [6] which is presented for the wave equation and applicable to the Schrödinger equation with no potential. Without loss of generality, one can consider initial data such that $\left\|\partial_{t} u(0, x)\right\|_{L^{2}(\Omega)}=1$. Then, let

$$
\Sigma_{T}=\frac{1}{T} \frac{G_{T}\left(\chi_{\omega}\right)}{\left\|\partial_{t} u(0, x)\right\|_{L^{2}(\Omega)}}=\frac{1}{T} G_{T}\left(\chi_{\omega}\right)
$$

and

$$
y_{j}(t, x)=i \lambda_{j} c_{j} \exp \left(i \lambda_{j} t\right) \phi_{j}(x) .
$$

Then, $\Sigma_{T}$ can be expressed as

$$
\begin{aligned}
\Sigma_{T} & =\frac{1}{T} \int_{0}^{T} \int_{\omega}\left(\left|\sum_{j=1}^{N} y_{j}(t, x)\right|^{2}+\left|\sum_{k=N+1}^{\infty} y_{k}(t, x)\right|^{2}\right. \\
& \left.+2\left(\sum_{j=1}^{N} y_{j}(t, x) \sum_{k=N+1}^{\infty} \overline{y_{k}(t, x)}\right) d x d t\right) .
\end{aligned}
$$

Note that

$$
C_{\infty}^{V}\left(\chi_{\omega}\right)=\inf \lim _{T \rightarrow \infty} \Sigma_{T}
$$


Now, we use the assumption that the spectrum of $-\Delta+V$ consists of simple eigenvalues to prove the following result.

Lemma 11. The following equation holds:

$$
\begin{gathered}
\lim _{T \rightarrow \infty} \frac{1}{T} \int_{0}^{T} \int_{\omega}\left|\sum_{j=1}^{N} y_{j}(t, x)\right|^{2} d x d t \\
=\sum_{j=1}^{N} \lambda_{j}^{2}\left|c_{j}\right|^{2} \int_{\omega} \phi_{j}^{2}(x) d x .
\end{gathered}
$$

Because the sum is finite, one can invert the inf and the limit. We have

$$
\begin{aligned}
& \frac{1}{T} \int_{0}^{T} \int_{\omega}\left|\sum_{j=1}^{N} y_{j}(t, x)\right|^{2} d x d t= \\
& \frac{1}{T} \sum_{j=1}^{N} \lambda_{j}^{2} \alpha_{j j} \int_{\omega} \phi_{j}^{2}(x) d x \\
& \quad+\frac{1}{T} \sum_{j=1}^{N} \sum_{k=1, k \neq j}^{N} \lambda_{j} \lambda_{k} c_{j} \overline{\mathcal{c}_{k}} \alpha_{j k} \int_{\omega} \phi_{j}(x) \overline{\phi_{k}(x)} d x,
\end{aligned}
$$

where $\alpha_{j k}$ was given previously by (14). Formula (14) gives

$$
\lim _{T \rightarrow \infty} \frac{\alpha_{j j}}{T}=1
$$

for every $j \in \mathbb{N}$. We note that

$$
\left|\alpha_{j k}\right| \leq \frac{\sqrt{2}}{\left|\lambda_{j}-\lambda_{k}\right|} \leq \frac{\sqrt{2}}{b_{1}}
$$

due to the fact that $|\exp (i \theta)-1|^{2}=(1-\cos \theta)^{2}+\sin ^{2} \theta$, for all $\theta \in \mathbb{R}$ and Theorem 9. We now estimate the remainder terms of (45):

$$
R=\frac{1}{T} \int_{0}^{T} \int_{\omega}\left|\sum_{j=N+1}^{\infty} y_{j}(t, x)\right|^{2} d x d t
$$

and

$$
\delta=\frac{1}{T}\left(\int_{0}^{T} \int_{\omega} \sum_{j=1}^{N} y_{j}(t, x) \sum_{k=N+1}^{\infty} \overline{y_{k}(t, x)} d x d t\right) .
$$

Using the fact that $\phi_{j}^{\prime} s$ form a Hilbert basis,

$$
\begin{aligned}
& R \leq \frac{1}{T} \int_{0}^{T} \int_{\Omega}\left|\sum_{j=N+1}^{\infty} y_{j}(t, x)\right|^{2} d x d t= \\
& \frac{1}{T} \sum_{j=N+1}^{\infty} \int_{0}^{T} \lambda_{j}^{2}\left|c_{j} \exp \left(i \lambda_{j} t\right)\right|^{2} d t=\frac{1}{T} \sum_{j=N+1}^{\infty} T \lambda_{j}^{2}\left|c_{j}\right|^{2} \\
&=\sum_{j=N+1}^{\infty} \lambda_{j}^{2}\left|c_{j}\right|^{2} .
\end{aligned}
$$

To bound $\delta$,

$$
\begin{aligned}
T|\delta| & =\left|\sum_{j=1}^{N} \sum_{k=N+1}^{\infty} \lambda_{j} c_{j} \lambda_{k} \overline{c_{k}} \alpha_{j k}\right| \\
& \leq \sum_{j=1}^{\infty}\left(\lambda_{j} c_{j}\right)^{2}\left(\sum_{k=N+1}^{\infty} \lambda_{k}^{2} c_{k}^{2} \alpha_{j k}^{2}\right) \\
& \leq \sum_{k=N+1}^{\infty}\left(\max _{j} \alpha_{j k} \lambda_{k} c_{k}\right)^{2},
\end{aligned}
$$

whenever the normalization $\sum_{j}\left(\lambda_{j} c_{j}\right)^{2}=1$ is used. By Parseval's theorem, since $u, \partial_{t}^{2} u \in L^{2}(\Omega)$, for every $\varepsilon>0$, there exists an $N \geq N(\varepsilon)$ such that

$$
\sum_{j=N+1}^{\infty} \lambda_{j}^{2}\left|c_{j}\right|^{2} \leq \varepsilon
$$

We conclude that, for sufficiently large $N$,

$$
|R+2 \delta| \leq \varepsilon\left(1+\frac{4}{T b_{1}}\right)
$$

Since $\varepsilon$ was arbitrary and $T \longrightarrow \infty$, the theorem is proved. The corollary follows since, due to the assumption that $\left\|\partial_{t} u(0, x)\right\|_{L^{2}(\Omega)}=1$, we have

$$
\inf _{\sum_{j=1}^{N} \lambda_{j}^{2}\left|c_{j}\right|^{2}=1} \sum_{j=1}^{N} \lambda_{j}^{2}\left|c_{j}\right|^{2} \int_{\omega} \phi_{j}^{2}(x) d x=\inf _{1, \ldots, N} \int_{\omega} \phi_{j}^{2}(x) d x
$$

Note that, in the case of nonsimple eigenvalues, one can group the diagonal terms to obtain the desired result. This proves Theorem 2 and Corollary 3.

\section{Basic Perturbation Theory}

In this section, we give an explicit example of how to calculate the eigenvalues and eigenvectors of the perturbed operator $H=-\Delta+\varepsilon V_{0}$, with simple eigenvalues $\lambda_{n}$. (Recall that this means the eigenvalues have multiplicity 1.) In the next section, more advanced results from [29] will be used to analyze the error terms.

Let $H_{0}=-\Delta$ denote the standard Laplacian with eigenvalues $\lambda_{n 0}$. There exists a corresponding basis $\phi_{n 0}(x)$ such that

$$
-\Delta \phi_{n 0}(x)=\lambda_{n 0} \phi_{n 0}(x)
$$

The following lemma relates the eigenvalues and eigenvectors of $H$ to those of $H_{0}$.

Lemma 12. The eigenvalues to $H$ are given by

$$
\lambda_{n}=\lambda_{n 0}+\varepsilon\left(\frac{\int V_{0}(x) \phi_{n 0}^{2}(x) d x}{\int \phi_{n 0}^{2}(x) d x}\right)+\mathcal{O}\left(\varepsilon^{2}\right) .
$$


The eigenfunctions to $H$ are given by

$$
\begin{aligned}
\phi_{n}(x)= & \phi_{n 0}(x) \\
& +\varepsilon\left(\sum_{n \neq m}\left(\frac{\int \phi_{n 0} \overline{V_{0} \phi_{m 0}} d x}{\lambda_{n 0}-\lambda_{m 0}}\right) \phi_{m 0}(x)\right) \\
& +\mathcal{O}\left(\varepsilon^{2}\right) .
\end{aligned}
$$

Here the $\mathcal{O}$ terms are uniform in $n$ depending on $\Omega$ and the $L^{\infty}(\Omega)$ norm of $V_{0}$. In particular we have that

$$
\left\|\phi_{n}(x)-\phi_{n 0}(x)\right\|_{L^{2}(\Omega)} \leq \varepsilon C_{2}\left(V_{0}, \Omega\right)\left\|\phi_{n 0}(x)\right\|_{L^{2}(\Omega)}
$$

and

$$
\begin{aligned}
& \| \phi_{n}(x)-\phi_{n 0}(x) \\
& \quad-\varepsilon\left(\sum_{n \neq m}\left(\frac{\int \phi_{n 0} \overline{V_{0} \phi_{m 0}} d x}{\lambda_{n 0}-\lambda_{m 0}}\right) \phi_{m 0}(x)\right) \|_{L^{2}(\Omega)} \\
& \quad \leq \varepsilon^{2} C_{3}\left(V_{0}, \Omega\right)\left\|\phi_{n 0}(x)\right\|_{L^{2}(\Omega)}
\end{aligned}
$$

where $C_{2}\left(V_{0}, \Omega\right), C_{3}\left(V_{0}, \Omega\right)$ depend only on the geometry of $\Omega$ and the $L^{\infty}(\Omega)$ norm of $V_{0}$.

We do not prove the Lemma here; it is a result of [29] (see equation (II-3.39) in Example 3.6, where the constant is given explicitly); we only give an idea of why it is true. One will see that the results in the next section are more general. If we make the approximation

$$
\begin{aligned}
\phi_{n}(x) & =\phi_{n 0}(x)+\varepsilon \phi_{n 1}(x)+\varepsilon^{2} \phi_{n 2}(x)+\cdots \\
\lambda_{n} & =\lambda_{n 0}+\varepsilon \lambda_{n 1}+\varepsilon^{2} \lambda_{n 2}+\cdots,
\end{aligned}
$$

then it follows by substitution that

$$
\begin{gathered}
\left(-\Delta+\varepsilon V_{0}(x)\right)\left(\phi_{n 0}(x)+\varepsilon \phi_{n 1}(x)+\mathcal{O}\left(\varepsilon^{2}\right)\right)= \\
\left(\lambda_{n 0}+\varepsilon \lambda_{n 1}+\mathcal{O}\left(\varepsilon^{2}\right)\right)\left(\phi_{n 0}+\varepsilon \phi_{n 1}(x)+\mathcal{O}\left(\varepsilon^{2}\right)\right) .
\end{gathered}
$$

Equating the leading order terms,

$$
\left(-\Delta-\lambda_{n 0}\right) \phi_{n 0}(x)=0 .
$$

At order $\varepsilon$, we have

$$
\left(-\Delta-\lambda_{n 0}\right) \phi_{n 1}+\left(V_{0}(x)-\lambda_{n 1}\right) \phi_{n 0}(x)=0 .
$$

The desired result for computing the first terms follows by taking the inner product of (68) with $\phi_{j 0}$ for $j \neq n$. We have to have a way of encoding this inductive process of matching up the terms. In the next section we introduce the operators $S$ and $P$ which allow us to do just that. The terms are computed for $L^{2}(\Omega)$ eigenfunctions, but the analysis is more sophisticated because, when computing the result of the matching over $L^{2}(\omega)$, one loses the orthogonality of the eigenfunctions over the region of integration.

We have the following example of an operator with simple eigenvalues.
Example 13. We consider the eigenvalue problem with $\alpha>1$

$$
\begin{aligned}
-u_{\varepsilon}^{\prime \prime}+\varepsilon x^{-2 \alpha} u_{\varepsilon} & =\lambda_{\varepsilon} u_{\varepsilon} \\
u_{\varepsilon}(0) & =u_{\varepsilon}(1)=0
\end{aligned}
$$

the unperturbed problem is

$$
\begin{aligned}
& -u^{\prime \prime}=\lambda u \\
& u(0)=u(1)=0
\end{aligned}
$$

with simple eigenvalues $\lambda=n^{2} \pi^{2}$, with $n=1,2,3 \cdots$ with corresponding normalized eigenfunctions $u=2^{1 / 2} \sin (n \pi x), n=1,2, \ldots$ The quadratic form associated with the potential $Q_{1}(f)$ with domain $\left\{f \in L^{2}(0,1): x^{-\alpha} f \in L^{2}(0,1)\right\} \subset H_{0}^{1}(\Omega)$ is closed in $L^{2}(0,1)$. The unperturbed operator is stable with respect to perturbations [29]. This is the assumption on both of the main theorems (Theorems 4 and 5). This example is from [30]. Stability of $\lambda$ for the unperturbed problem means that, for $\varepsilon$ sufficiently small, the intersection of any isolating interval for $\lambda$ and the spectrum of the perturbed operator consists only of simple eigenvalues. The unperturbed/perturbed operator pair here satisfies the criterion of Theorem 5.1.12 in [29] for stability which holds provided the left hand side (86) is smaller than $1 / 2$, which is true for sufficiently small $\varepsilon$. This also applies to the first numerical example in the Appendix. Usually stability is automatically satisfied when $-\Delta$ has simple spectrum and $\varepsilon_{0}$ is sufficiently small, c.f. Lemma 2.1 in [31].

As a general remark on the example and computations above, the difficulty lies in quantifying the error terms which are usually formulated in the sense of $L^{2}(\Omega)$ not $L^{2}(\omega)$, which is why the next section is required.

We have the following result for more general Riemannian metrics which shows that the assumption of simple spectrum in our case covers generic domains $\Omega$. Symmetry usually destroys the assumption of spectral simplicity, c.f. [32], and this is also discussed in Section 7.

Theorem 14 (see [33] and [32]). Let $\mathscr{M}$ be a compact manifold of dimension greater than 1 and $\mathscr{C}$ a conformal class of Riemannian metrics of fixed volume on $\mathscr{M}$. Given $k \geq 1$ and $d \geq 2$, the subset of $\mathscr{C}$ of metrics for which the $k^{\text {th }}$ eigenspace is of dimension $d$ is a submanifold of codimension of at least 1. In particular, the subset of $\mathscr{C}$ of metrics admitting a nonsimple eigenvalue of the Laplacian is a countable union of submanifolds of codimension of at least 1.

This theorem asserts that for a given compact manifold $\mathscr{M}$ "most" Riemannian metrics $g$ on $\mathscr{M}$ are simple, meaning the eigenspace of the Laplace operator $\Delta_{g}$ is one-dimensional and that this set is pathwise connected. The proof naturally remains true for $0^{\text {th }}$ order perturbations, like the ones we have here, c.f. [31]. We leave the question of what happens to the observability constants for metric perturbations to future work. 


\section{Advanced Perturbation Theory}

In this section, we elaborate on advanced perturbation theory for a better understanding of the results derived in the paper. Let $X$ be an arbitrary Hilbert space, as in [29], and $R(A)$ be the range of the bounded operator $A$. The monograph [29] by Kato computes perturbation theory results for generic bounded operators $A$, and since our operator satisfies the conditions in [29] for a Type (A) holomorphic operator in the parameter $\varepsilon$ (Theorem 2.6 of [29]), the perturbation theory derived in the book applies.

Let $P$ be the projection operator and $\lambda$ be one of the eigenvalues of $H_{0}=-\Delta, H=-\Delta+\varepsilon V_{0}=-\Delta+V$, and let $\lambda_{k}, P_{k}, k=1,2, \ldots$, be the eigenvalues and eigenprojections of $H_{0}=-\Delta$ different from $\lambda$ and $P$ under consideration. Let $\left\{x_{1}, . ., x_{m}\right\}$ denote a basis of $M=R(P)$ and $\left\{x_{k 1}, . ., x_{k m_{k}}\right\}$ denote a basis of $M_{k}=R\left(P_{k}\right)$ for each $k$. The union of the vectors $x_{j}$ and $x_{k j}$ forms a basis of $X$ consisting of eigenvectors of $H_{0}=-\Delta$ and is adapted to $X=M \oplus M_{1} \oplus \cdots$ of $X$. The adjoint basis of $X^{\star}$ is adapted to $X^{\star}=M^{\star} \oplus M_{1}^{\star} \oplus \cdots$, where $M^{\star}=R\left(P^{\star}\right), M_{1}^{\star}=R\left(P_{1}^{\star}\right)$, etc. Let $\left\{e_{1}, \ldots, e_{m}\right\}$ denote the adjoint basis of $M^{*}$ and $\left\{e_{k 1}, \ldots, e_{k m_{h}}\right\}$ denote the basis of $M_{k}^{*}$ for $k=1,2, \ldots$.

For any $u \in X$,

$$
\begin{aligned}
P u & =\sum_{j=1}^{m}\left\langle u, e_{j}\right\rangle x_{j} \\
P_{k} u & =\sum_{j=1}^{m_{k}}\left\langle u, e_{k j}\right\rangle x_{k j}, \quad \forall k=1,2 \ldots
\end{aligned}
$$

We define the operator $S$ as the value of the reduced resolvent of $H_{0}=-\Delta$, such that $S P=P S=0$, and $\left(H_{0}-\lambda\right) S=S\left(H_{0}-\right.$ $\lambda)=1-P . P_{k}^{\prime} s$ are the orthogonal projections such that

$$
P=\sum_{k=1}^{\infty} P_{k}
$$

and moreover, by definition, $P^{2}=P$. For $\lambda$ in our particular eigenspace, it follows that one can write the operator $S$ explicitly as

$$
S u=\sum_{k}\left(\lambda_{k}-\lambda\right)^{-1} P_{k} u=\sum_{k, j}\left(\lambda_{k}-\lambda\right)^{-1}\left\langle u, e_{k j}\right\rangle x_{k j} .
$$

using the definitions (I-5.32) and Section II.2 in [29]. If we expand $\lambda_{\varepsilon}$, which is an eigenvalue of $H$, in a perturbation series as

$$
\lambda_{\varepsilon}=\lambda+\varepsilon \widehat{\lambda}^{1}+\varepsilon^{2} \widehat{\lambda}^{2}+\cdots,
$$

one obtains the following expressions for the expansions of the eigenvalues $\hat{\lambda}^{n}$ (II-(2.35) [29]):

$$
\begin{aligned}
& \hat{\lambda}^{1}=\frac{1}{m} \sum_{j}\left\langle V_{0} x_{j}, e_{j}\right\rangle . \\
& \hat{\lambda}^{2}=-\frac{1}{m} \sum_{i, j, k}\left(\lambda_{k}-\lambda\right)^{-1}\left\langle V_{0} x_{1}, e_{k j}\right\rangle\left\langle V_{0} x_{k j}, e_{i}\right\rangle .
\end{aligned}
$$

Suppose that the eigenvalue of $\lambda$ of $H_{0}$ is simple, implying that $m=1$. To derive an expansion for a particular eigenvector eigenvalue pair, one can set $x_{1}=\phi_{j 0}(x)$ and $e_{1}=\bar{\phi}_{j 0}(x)$ as in the last section. (Now $j$ just refers to the index of the eigenfunction, a distinct index from the one above.) The operators $P_{j}$ and $S_{j}$ can be written as

$$
\begin{aligned}
P_{j} u & =\left\langle u, \phi_{j 0}\right\rangle_{L^{2}(\Omega)} \phi_{j 0}, \\
S u & =\sum_{j \neq k} \frac{P_{k} u}{\lambda_{j 0}-\lambda_{k 0}} .
\end{aligned}
$$

This substitution compares immediately to the results in the previous section for the expansion of the eigenvalues (61). Now we describe a more advanced decomposition of the eigenvectors.

Assuming for simplicity that $m=1$, a convenient form of the eigenvector $\phi_{j}$ of $H=-\Delta+\varepsilon V_{0}$ corresponding to the eigenvalue $\lambda_{\varepsilon}$ is given by

$$
\phi_{j}=\left\langle P_{j}(\varepsilon) \phi_{j 0}, \overline{\phi_{j 0}}\right\rangle_{L^{2}(\Omega)}^{-1} P_{j}(\varepsilon) \phi_{j 0},
$$

where $\phi_{j 0}$ is the unperturbed operator of $H_{0}$ for the eigenvalue $\lambda$ and $\bar{\phi}_{j 0}$ is the eigenvector of the adjoint operator $H_{0}^{\star} . P_{j}(\varepsilon)$ is the projection onto the $j^{\text {th }}$ eigenspace of $H$. The assumption of stability here is used in a hidden way as we want to make sure the projection onto the eigenspace is well-defined. In particular the projection is defined as the integral of the resolvent over an interval containing only one eigenvalue. As such, in order for the projection to be well-defined, the eigenvalue needs to be sufficiently isolated, whence the assumption of simplicity in a perturbed neighbourhood of $-\Delta$. We refer the reader to Theorem 5.1.12 in [29] and Lemma 2.1 in [31] for a precise description of $\varepsilon_{0}$, the threshold required. In the case of nonsimple eigenvalues the representation above would depend on more than one $\phi_{j 0}$, which would be difficult to analyze. We suppress the subscript $j$ in the operators $P$ and $S$ where it is understood. This gives rise to the following normalization conditions:

$$
\begin{aligned}
\left\langle\phi_{j}, \overline{\phi_{j 0}}\right\rangle_{L^{2}(\Omega)} & =1, \\
\left\langle\phi_{j}-\phi_{j 0}, \overline{\phi_{j 0}}\right\rangle_{L^{2}(\Omega)} & =0, \\
P\left(\phi_{j}-\phi_{j 0}\right) & =0 .
\end{aligned}
$$

The relation $\left(H-\lambda_{\varepsilon}\right) \phi_{j}=0$ can be rewritten as

$$
\left(H_{0}-\lambda\right)\left(\phi_{j}-\phi_{j 0}\right)+\left(V-\lambda_{\varepsilon}+\lambda\right) \phi_{j}=0,
$$

where $A=H-H_{0}=\varepsilon V_{0}(x)=V$. Multiplying (80) from the left hand side by $S$ and noting that $S\left(H_{0}-\lambda\right)=1-P$,

$$
\phi_{j}-\phi_{j 0}+S\left[V-\lambda_{\varepsilon}+\lambda\right] \phi_{j}=0 .
$$

Moreover, as $S \phi_{j 0}=0$ and writing $\phi_{j}=\phi_{j}-\phi_{j 0}+\phi_{j 0}$ in the last term above, one gets

$$
\begin{aligned}
\phi_{j}-\phi_{j 0} & =-\left(1+S\left(V-\lambda_{\varepsilon}+\lambda\right)\right)^{-1} S V \phi_{j 0} \\
& =-S\left(1+V S-\left(\lambda_{\varepsilon}-\lambda\right) S_{\alpha}\right)^{-1} V \phi_{j 0},
\end{aligned}
$$


for sufficiently small $\varepsilon$, with $S_{\alpha}=S-\alpha P$, and $\alpha$ is an arbitrary scalar. Equation (82) is formula (II-3.29) in [29].

One can then compute

$$
\begin{aligned}
& \left\langle\phi_{j}-\phi_{j 0}, \phi_{j 0}\right\rangle_{L^{2}(\omega)} \\
& \quad=\left\langle-S\left(1+V S-\left(\lambda_{\varepsilon}-\lambda\right) S_{\alpha}\right)^{-1} V \phi_{j 0}, \phi_{j 0}\right\rangle_{L^{2}(\omega)} .
\end{aligned}
$$

The asymptotics for the scalar $\lambda_{j}$ are well worked out for small $\varepsilon$. Let $q=\left\|V_{0} S\right\|, s_{0}=\|S\|, p=\left\|V_{0} P\right\|, s=\|S-\alpha P\|$ for any $\alpha$, where we use the operator norm. A subscript will denote the set over which the operator norm is taken.

For a linear operator $A$ acting on $H_{0}^{1}(\Omega)$, we let $\|A\|_{0}$ denote the norm

$$
\begin{aligned}
& \|A\|_{0} \\
& =\left\{\sup _{j}\left\langle A u, \phi_{j 0}\right\rangle \text { such that } u: \sup _{j}\left\langle u, \phi_{j 0}\right\rangle=1\right\} .
\end{aligned}
$$

Set $p, s, q$ to have norm $\|\cdot\|_{0}$ and define

$$
\Psi(\varepsilon)=\left((1-(p s+q) \varepsilon)^{2}-4 p s \varepsilon^{2}\right)^{1 / 2} .
$$

As a result

$$
\begin{aligned}
\left|\lambda_{j}-\lambda_{j 0}-\varepsilon \widehat{\lambda}^{1}\right| & =\left|\lambda_{\varepsilon}-\lambda-\varepsilon \widehat{\lambda}^{1}\right| \\
& \leq \frac{2 p q \varepsilon^{2}}{1-(p s+q) \varepsilon+\Psi(\varepsilon)}
\end{aligned}
$$

which is formula (II-3.18) in [29], with the norm $\|\cdot\|_{0}$. The expansion (83) derived above is given in section II and exercise II-3.16 in the monograph by Kato [29].

Now the difficulty comes in computing inner products of $\phi_{j}-\phi_{j 0}$ over the smaller sets $\omega$ where one loses the powerful orthogonality conditions. We recall the following well-known Lemma on von Neumann series.

Lemma 15. Let $A: X \longrightarrow X$ be a linear operator on the Banach space $X$. We then have

$$
\sum_{j=0}^{\infty} A^{j} u=(I d-A)^{-1} u \quad \forall u \in X
$$

provided

$$
\left\|A^{j} u\right\|_{X} \leq \delta_{1}^{j}\|u\|_{X} \quad \forall j \in \mathbb{N}
$$

with $\delta_{1} \in(0,1 / 2)$.

\section{c.f. Lemma 2.1 in [34].}

In order to compute (83), we want to use Lemma 15 to essentially find a convergent von-Neumann series for

$$
\left(1+V S-\left(\lambda_{\varepsilon}-\lambda\right) S_{\alpha}\right)^{-1}
$$

with (86) so that we may obtain precise bounds on the rate of decay of the inner products $\left\langle\phi_{j}-\phi_{j 0}, \phi_{j 0}\right\rangle_{L^{2}(\omega)}$. These arguments are rather delicate as we are not integrating over the whole $\Omega$. We let $M_{0}$ denote a generic constant that depends on the volume of $\Omega$ and $\left\|V_{0}\right\|_{L^{\infty}(\Omega)}$ We state the four necessary Lemmas first, followed by their technical proofs to see how the pieces fit together to allow us to use Lemma 15 by examining each term in the series expansion to bound (89).

Lemma 16. With no assumptions on the support of the potential, we have the following estimate for $u_{\omega} \in L^{2}(\omega)$ with $\operatorname{supp}\left(u_{\omega}\right) \subseteq \omega$

$$
\begin{aligned}
& \left|\left\langle V\left(S u_{\omega}\right), u_{\omega}\right\rangle_{L^{2}(\omega)}\right| \\
& \quad=\left|\sum_{j \neq k} \frac{\left\langle u_{\omega}, \phi_{k 0}\right\rangle_{L^{2}(\Omega)}\left\langle V \phi_{k 0}, u_{\omega}\right\rangle_{L^{2}(\omega)}}{\lambda_{j 0}-\lambda_{k 0}}\right| \\
& \quad \leq \varepsilon M_{0}\left\|u_{\omega}\right\|_{L^{2}(\omega)}^{2} .
\end{aligned}
$$

Let $A_{v}$ be the linear operator defined as multiplication by

$$
\left(\left(\lambda_{j 0}-\lambda_{j}\right)-V\right)
$$

Lemma 17. With no assumptions on the support of the potential, we have the following estimate for $u_{\omega} \in L^{2}(\omega)$ with $\operatorname{supp}\left(u_{\omega}\right) \subseteq \omega$ for all $N \geq 0, N \in \mathbb{N}$,

$$
\left|\left\langle\left(A_{v} S\right)^{N}\left(u_{\omega}\right), u_{\omega}\right\rangle_{L^{2}(\omega)}\right| \leq\left(\varepsilon M_{0}\right)^{N}\left\|u_{\omega}\right\|_{L^{2}(\omega)}^{2} .
$$

Lemma 18. If $\operatorname{supp}\left(V_{0}\right) \subset \omega$ and $\alpha \in(0,1)$, then the operator $S\left(1-A_{v} S-\alpha\left(\lambda_{j}-\lambda_{j 0}\right) P\right)^{-1} V$ is bounded $L^{2}(\Omega) \longmapsto L^{2}(\omega)$.

Lemma 19. There is a choice of $\varepsilon$ sufficiently small, such that, for all $\delta \in(0,1 / 2)$, the following inequality holds:

$$
\begin{aligned}
& \left|\left\langle\phi_{j 0}-\phi_{j}, \phi_{j 0}\right\rangle_{L^{2}(\omega)}\right| \\
& \quad=\left|\left\langle S\left(1+V S-\left(\lambda_{\varepsilon}-\lambda\right) S_{\alpha}\right)^{-1}\left(V \phi_{j 0}\right), \phi_{j 0}\right\rangle_{L^{2}(\omega)}\right| \\
& \quad \leq \delta\left\|\phi_{j 0}\right\|_{L^{2}(\omega)}^{2} .
\end{aligned}
$$

Proof of Lemma 16. By the Cauchy Schwartz inequality, we have

$$
\begin{aligned}
& \left|\left\langle V S u_{\omega}, u_{\omega}\right\rangle_{L^{2}(\omega)}\right| \leq \sum_{j \neq k} \frac{\left|\left\langle u_{\omega}, \phi_{j}\right\rangle_{L^{2}(\Omega)}\left\langle u_{\omega}, V \phi_{j}\right\rangle_{L^{2}(\omega)}\right|}{\left|\lambda_{j}-\lambda_{k}\right|} \\
& \quad \leq\left(\sum_{j \neq k} \frac{\left|\left\langle u_{\omega}, \phi_{j}\right\rangle_{L^{2}(\Omega)}\right|^{2}}{\left|\lambda_{j}-\lambda_{k}\right|^{2}}\right)^{1 / 2} \\
& \quad \cdot\left(\sum_{j \neq k}\left|\left\langle u_{\omega}, V \phi_{j}\right\rangle_{L^{2}(\omega)}\right|^{2}\right)^{1 / 2}
\end{aligned}
$$

We know from Theorem 3.4 from [28] that

$$
\left|\lambda_{j 0}-\lambda_{k 0}\right|>C, \quad j \neq k
$$


where $C$ depends on $\Omega$ independent of the index set. The constant exists because all of the eigenvalues are simple and isolated. However in practice for dimensions higher than 2 the size of $C$ may be difficult to ascertain. The desired result follows immediately from Parseval's theorem, noting that $u_{\omega}$ and $V u_{\omega}$ are $L^{2}(\Omega)$ functions. We remark that this is where we use the assumption supp $V \subset \omega$ later for the main proof as the inner product $\left\langle u_{\omega}, \phi_{j}>\right.$ is over $L^{2}(\Omega)$ which cannot be bounded by $\left\|u_{\omega}\right\|_{L^{2}(\Omega)}$ unless $u_{\omega}$ has compact support in $\omega$.

Proof of Lemma 17. The bound for this inner product is constructed inductively as

$$
\begin{aligned}
& \left(A_{v} S\right)^{N}\left(u_{\omega}\right)=\sum_{m_{0} \neq j} \frac{\left\langle u_{\omega}, \phi_{m_{0}}\right\rangle_{L^{2}(\Omega)}}{\lambda_{j 0}-\lambda_{m_{0}}} \\
& \cdot \sum_{m_{1} \neq j} \frac{\left\langle A_{v} \phi_{m 0}, \phi_{m_{1}}\right\rangle_{L^{2}(\Omega)}}{\lambda_{j 0}-\lambda_{m_{1}}} \\
& \ldots \sum_{m_{N-1} \neq j} \frac{\left\langle A_{v} \phi_{m_{N-2}}, \phi_{m_{N-1}}\right\rangle_{L^{2}(\Omega)}}{\lambda_{j 0}-\lambda_{m_{N-1}}} A_{v} \phi_{m_{N-1}}
\end{aligned}
$$

We then use the proof of Lemma 16, but with (94) applied to each of the cross terms

$$
\sum_{m_{i-1} \neq j} \frac{\left\langle A_{v} \phi_{m_{i-2}}, \phi_{m_{i-1}}\right\rangle_{L^{2}(\Omega)}}{\lambda_{j 0}-\lambda_{m_{i-1}}} \quad i=2, \ldots, N
$$

to reach the desired conclusion, noting that $\left\|A_{v}\right\|_{L^{\infty}(\Omega)}$ is almost equivalent to $\|V\|_{L^{\infty}(\Omega)}$. Alternatively we know, for bounded operators $A, B$ with $A: X \longrightarrow X, B: X \longrightarrow X, X$ a Hilbert space, that $\|A B\|_{o p} \leq\|A\|_{o p}\|B\|_{o p}$ which when applied to $A=V$ and $B=S$ from the previous lemma gives the result as well.

Proof of Lemma 18. The mapping $A_{v} S$ satisfies all the properties of Lemma 15 with $X$ the space $L^{2}(\Omega)$ restricted to the functions with compact support in $\omega$, by Lemma 17 . The space $X$ is $L_{0}^{2}(\omega)$, which is a Hilbert space (although most people are more familiar with $\left.H_{0}^{1}(\omega)\right)$. Therefore since $V u \in X$, for all $u \in L^{2}(\Omega)$, we are done.

Proof of Lemma 19. The inner product using (83) and Lemmas 17 and 15 with $A_{v} S+\alpha\left(\lambda_{j}-\lambda_{j 0}\right) P$ and $\delta_{1}=\varepsilon M_{0}$ is bounded as

$$
\begin{gathered}
\left\|\phi_{j 0}\right\|_{L^{2}(\omega)}^{2}\left(\left(\varepsilon M_{0}\right)+\left(\varepsilon M_{0}\right)^{2}+\cdots\right) \\
=\frac{\varepsilon M_{0}}{1-\varepsilon M_{0}}\left\|\phi_{j 0}\right\|_{L^{2}(\omega)}^{2} .
\end{gathered}
$$

If $\varepsilon M_{0}$ is chosen to be sufficiently small, one obtains

$$
\frac{\varepsilon M_{0}}{1-\varepsilon M_{0}}<\delta
$$

with $\delta \in(0,1 / 2)$, implying $\varepsilon<\delta / 2 M_{0}$. We use the fact supp $V_{0} \subset \omega$, which makes the function $V \phi_{j} \forall j$ have support in $\omega$.
Remark 20. Some of the analysis could be extended to the case of nonsimple eigenvalues and other Hermitian operators using perturbation theory found in [29], but we focus on simple eigenvalues for ease and clarity.

\section{Proof of Theorems 4 and 5 for Convergence Estimates}

Proof of Theorem 4. We recall that eigenfunctions of $-\Delta$ and $-\Delta+\varepsilon V_{0}$ ( $\varepsilon$ sufficiently small) with Dirichlet boundary conditions are real-analytic in $\Omega$. We can then view $\int_{\omega} \phi_{j}^{2}(x) d x=$ $f_{V}(j)$ as a function of $\mathbb{N}$ taking values in $(0,1]$ and similarly for $\int_{\omega} \phi_{j 0}^{2}(x) d x=f(j)$. We only need to show that the following inequality is true for some order terms independent of the index $j$ :

$$
\int_{\omega} \phi_{j}^{2}(x) d x=(1+\mathcal{O}(\epsilon)) \int_{\omega} \phi_{j 0}^{2}(x) d x .
$$

These terms will bound the deviation from the original constant when including the potential term, and taking inf ${ }_{j}$ of the inequality gives the desired result. We need control over the order $\epsilon$ terms and show they are uniformly bounded, independent of $\phi_{j 0}$; e.g., the order terms are smaller than $1 / 2$ for sufficiently small $\epsilon$. If we can show this inequality, we will arrive at

$$
\frac{1}{2} f_{V}(j) \leq f(j) \leq \frac{3}{2} f_{V}(j) .
$$

Taking the infimum over $j$ gives the desired conclusion.

By the perturbation theory estimates in Section 6, in Lemmas 17 and 19, by using the Lemma 15, the terms

$$
2\left|\left\langle\phi_{j 0}-\phi_{j}, \phi_{j 0}\right\rangle_{L^{2}(\omega)}\right|+\left\|\phi_{j 0}-\phi_{j}\right\|_{L^{2}(\omega)}^{2}
$$

which contribute to the order terms in (100) are bounded as in Lemma 19. Therefore, it suffices to pick $\varepsilon$ as in Lemma 19: $3 \delta<1 / 2$, in order to obtain (100), with $\mathcal{O}(\epsilon)$ terms less than $1 / 2$.

We now take a moment to remark on why the assumption of simplicity in Theorem 4 is spectrally sharp, by outlining a counter-example from the details of [8]. In Remark 2.2 of [8], on the sphere $\mathbb{S}^{2}$ they construct a potential of arbitrarily small support and size and an open set $\omega \subset \mathbb{S}^{2}$ such that

$$
\begin{aligned}
& \inf \left\{\int_{\omega} \phi^{2}(x) d x \phi\right. \text { eigenf unction of } \\
& \left.-\Delta \text { s.t. }\|\phi\|_{L^{2}\left(\mathbb{S}^{2}\right)}=1\right\}=0
\end{aligned}
$$

however

$$
\begin{aligned}
& \inf \left\{\int_{\omega} \psi^{2}(x) d x \psi \text { eigenf unction of }-\Delta\right. \\
& \left.\quad+V \text { s.t. }\|\psi\|_{L^{2}\left(\mathbb{S}^{2}\right)}=1\right\}>0
\end{aligned}
$$


One can take this a step further and construct a sequence of normalized eigenfunctions of $-\Delta$ such that $\phi_{j}$ belongs to the eigenspace associated with the eigenvalue $j(j+1)$ in such a way that

$$
\lim _{j \rightarrow \infty} \int_{\omega} \phi_{j}^{2}(x) d x=0
$$

as in [9]. One can complete this sequence to obtain an orthonormal basis of $L^{2}\left(\mathbb{S}^{2}\right)$ consisting of eigenfunctions of $-\Delta$ such that $J^{0}\left(\chi_{\omega}\right)=0$. On the other hand, $J^{V}\left(\chi_{\omega}\right)>0$. In this particular case where the spectrum is nonsimple, one cannot obtain the strong iff statement in Theorem 4 because the representation for the perturbed eigenfunctions in (78) depends on all of the $2 j+1$ eigenfunctions of $-\Delta$ associated with each eigenvalue $j(j+1)$, introducing cross terms in Lemmas 16 and 17 which are computationally difficult to control. If the perturbation theory was carried out for this nonsimple case which is possible, then at most one could conclude that the constants are close.

Proof of Theorem 5. Let $\widetilde{\phi}=\phi_{j 0}-\phi_{j}$; then we can write for any $j$ and $a(x)$

$$
\begin{aligned}
\int_{\Omega} a(x) \phi_{j}^{2} d x= & \int_{\Omega} a(x) \phi_{j 0}^{2} d x \\
& +\int_{\Omega} a(x)\left(2 \phi_{j 0} \tilde{\phi}+\widetilde{\phi}^{2}\right) d x .
\end{aligned}
$$

As we have that using the normalisation condition $\left\|\phi_{j 0}\right\|_{L^{2}(\Omega)}^{2}=1$, and the "observation region" is the entirety of $\Omega$

$$
\begin{aligned}
& \left|\int_{\Omega} a(x)\left(2 \phi_{j 0} \tilde{\phi}+\widetilde{\phi}^{2}\right) d x\right| \leq 3\left|\int_{\Omega} a(x) \widetilde{\phi}^{2} d x\right| \\
& \quad \leq 3 L|\Omega| C_{2}^{2}\left(V_{0}, \Omega\right) \varepsilon^{2} .
\end{aligned}
$$

We obtain

$$
\begin{aligned}
& \left|\int_{\Omega} a(x) \phi_{j}^{2} d x-\int_{\Omega} a(x) \phi_{j 0}^{2} d x\right| \\
& \quad \leq 3 L|\Omega| C_{2}^{2}\left(V_{0}, \Omega\right) \varepsilon^{2}
\end{aligned}
$$

where the order terms are uniformly bounded where we have used Lemma 12. We set $C_{1}\left(V_{0}, \Omega\right)=3|\Omega| C_{2}^{2}\left(V_{0}, \Omega\right)$. Notice that the orthogonality relations imply that no additional regularity is needed on $V_{0}$. Without loss of generality assume $J_{\varepsilon}(a)-J(a)>0$; then we have that

$$
\begin{aligned}
& J_{\varepsilon}(a)+\inf _{j}\left(\int_{\Omega} a(x) \phi_{j 0}^{2} d x-\int_{\Omega} a(x) \phi_{j}^{2} d x\right) \\
& \leq J(a) .
\end{aligned}
$$

Rearranging, we obtain a bound on $J_{\varepsilon}(a)-J(a)$, depending on $|\Omega|$ and the $L^{\infty}(\Omega)$ norm of the potentials as desired, after using (107). Notice that this is probably the best control of the errors as $-\inf _{j}(A(j))=\sup _{j}(-A(j))$ for all functionals $A(j)$.
With out loss of generality we assume $\max _{a \in \bar{M}_{L}} J_{\varepsilon}(a)-$ $\max _{a \in \bar{M}_{L}} J(a)>0$, and we obtain

$$
\begin{aligned}
\max _{a \in \bar{M}_{L}} J_{\varepsilon}(a) & \leq \max _{a \in \bar{M}_{L}}\left(J_{\varepsilon}(a)-J(a)\right)+\max _{a \in \bar{M}_{L}} J(a) \\
& \leq C_{1}\left(V_{0}, \Omega\right) \varepsilon^{2}+\max _{a \in \bar{M}_{L}} J(a)
\end{aligned}
$$

with constant given to us by (107) and Lemma 19.

\section{Numerics and Examples}

This section presents the results of our numerical experiments. We examine the cases of the unit interval and the unit disk. The convergence issues for the functionals in question are discussed in the Appendix.

8.1. Interval $[0,1]$. The first experiment involved $\Omega=[0,1]$. The orthonormal eigenvectors of $-\Delta$ with Dirichlet boundary conditions on this domain are $f_{n}(x)=\sqrt{2} \sin (n \pi x)$, for $n=$ $1,2, \ldots$, with eigenvalues of $\lambda_{n}=\pi^{2} n^{2}$, for $n=1,2, \ldots$. The eigenvalues have multiplicity one.

Next, we calculate the eigenvectors and eigenvalues of $H=-\Delta+\varepsilon V_{0}$ on the unit interval with Dirichlet boundary conditions. According to perturbation theory of Section 5, the eigenvalues of operator $H$ are given by

$$
\lambda_{n}=\lambda_{n 0}+\varepsilon\left(\frac{\int V_{0}(x) \phi_{n 0}^{2}(x) d x}{\int \phi_{n 0}^{2}(x) d x}\right)+\mathcal{O}\left(\varepsilon^{2}\right) .
$$

The eigenfunctions of $H$ are given by

$$
\begin{aligned}
\phi_{n}(x)= & \phi_{n 0}(x) \\
& +\varepsilon\left(\sum_{n \neq m}\left(\frac{\int \phi_{n 0} \overline{V_{0} \phi_{m 0}} d x}{\lambda_{n 0}-\lambda_{m 0}}\right) \phi_{m 0}(x)\right) \\
& +\mathcal{O}\left(\varepsilon^{2}\right) .
\end{aligned}
$$

In our case, we use the potential

$$
V_{0}=x^{2} \chi_{[0.5-\delta, 0.5+\delta]},
$$

where $\delta$ is a parameter $\in[0,0.5]$.

Matlab was used to code the experiments. The integration of functions with explicit formulas was performed using the integral function in Matlab. We used a mesh size of about 1000 equal increments. The first two hundred eigenfunctions were calculated.

Next, we consider the problem of maximizing the functional $J_{N}^{V}\left(\chi_{\omega}\right)$,

$$
J_{N}^{V}\left(\chi_{\omega}\right)=\inf _{1 \leq j \leq N} \int_{\omega} \phi_{j}^{2}(x) d x
$$

over all subsets satisfying $|\omega|=L|\Omega|$, for some $L \in(0,1)$. A subset with this property is called the optimal set. According to Proposition 4.1 of [6], in the case of the $-\Delta$ operator, the 
TABLE 1: Value of $J_{N}^{V}\left(\chi_{[0,0.5]}\right)$.

\begin{tabular}{lccccc}
\hline$\varepsilon / \delta$ & 0.1 & 0.2. & 0.3. & 0.4 & 0.475. \\
\hline 0.01 & 0.499997124 & 0.499984760 & 0.499972504 & 0.499968543 & 0.499968340 \\
0.05 & 0.499985619 & 0.499923804 & 0.499862542 & 0.499842757 & 0.499841748 \\
0.1 & 0.499971238 & 0.499847620 & 0.499725137 & 0.499685621 & 0.499683617 \\
0.5 & 0.499856202 & 0.499238531 & 0.498627808 & 0.498432406 & 0.498422979 \\
1 & 0.499712437 & 0.498478145 & 0.497260919 & 0.496875569 & 0.496858189 \\
\hline
\end{tabular}

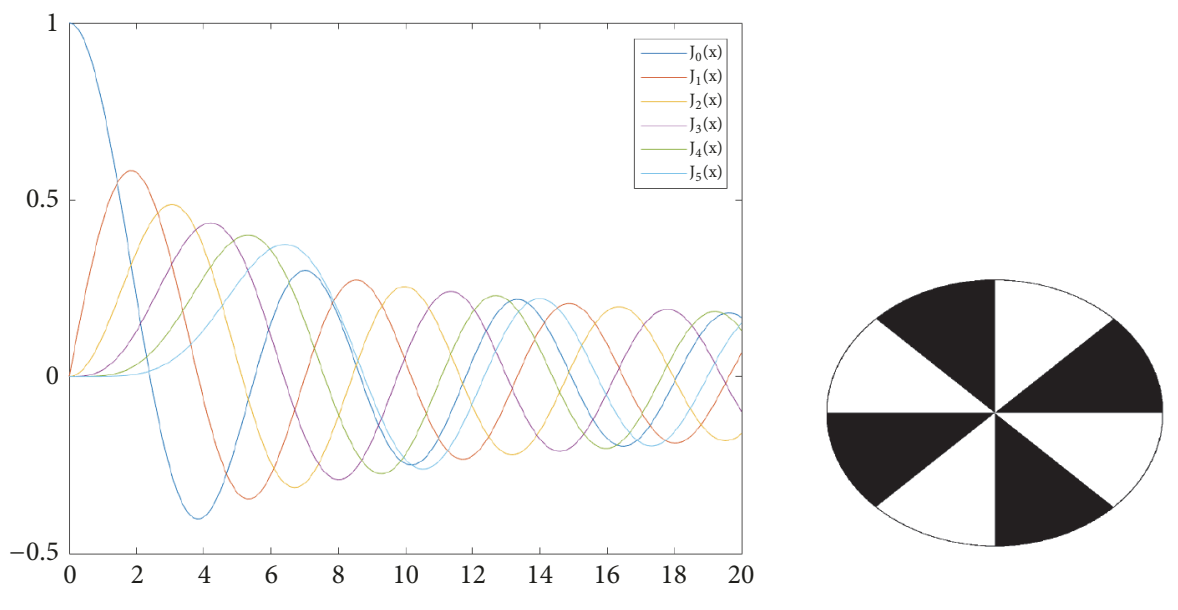

FIGURE 1: Graph of Bessel Functions and the region of integration.

supremum of $J\left(\chi_{\omega}\right)=\inf _{1 \leq j \leq N} \int_{\omega} \phi_{j 0}^{2}(x)$ over $\mathscr{M}_{L}$ is equal to $\mathrm{L}$. When $L=0.5$, the supremum is reached for all measurable subsets $\omega$ of $[0,1]$ satisfying $|\omega|=0.5|\Omega|$, such that $\omega$ and its symmetric image are complementary in $[0,1]$. Note that, for the $-\Delta$ operator, inf ${ }_{1 \leq j \leq N} \int_{[0,0.5]} \phi_{j 0}^{2}(x) d x=0.5$ since

$$
\int_{0}^{0.5} 2 \sin ^{2}(n \pi x) d x=0.5 . \quad \forall n=1,2,3, \ldots
$$

The more interesting case is the $H=-\Delta+\varepsilon V_{0}$ operator, and we address the question by using $L=0.5$ and computing $J_{N}^{V}\left(\chi_{\omega}\right)$ for subsets satisfying $|\omega|=0.5|\Omega|$ and the conditions of Proposition 4.1 of [6]. In particular, we present results for $\omega=[0,5]$ and $N=200$.

To calculate $J_{N}^{V}\left(\chi_{[0,0.5]}\right)$ for $H=-\Delta+\varepsilon V_{0}$, integration using the left point and 1000 equal increments in $[0,1]$ were used. The $\delta$ and $\varepsilon$ variables were varied as shown in Table 1. The values in the table show that, in all cases, the value of $J_{N}^{V}\left(\chi_{[0,0.5]}\right)$ is very close to 0.5 , which is the answer for the $-\Delta$ operator.

8.2. Unit Disk. The orthonormal eigenvectors of $-\Delta$ on a unit disk with Dirichlet boundary conditions are given by the triply indexed sequence

$$
\phi_{j k m 0}= \begin{cases}\frac{R_{j k}(r)}{\sqrt{2 \pi}}, & \text { if } j=0, \\ R_{j k}(r) Y_{j m}(\theta), & \text { if } j \geq 1,\end{cases}
$$

for $j=0,1,2, \ldots, k=1,2, \ldots$, and $m=1,2$, where $(r, \theta)$ are polar coordinates. Here, $Y_{j 1}(\theta)=(1 / \pi) \cos (j \theta), Y_{j 2}(\theta)=$ $(1 / \pi) \sin (j \theta)$, and

$$
R_{j k}(r)=\sqrt{2} \frac{J_{j}\left(z_{j k} r\right)}{\left|J_{j}^{\prime}\left(z_{j k}\right)\right|},
$$

where $J_{j}$ is the Bessel function of the first kind of order $j$ and $z_{j k}>0$ is the $k^{t h}$ zero of $J_{j}$. The eigenvalues are given by the double sequence of $-z_{j k}^{2}$. Their multiplicity is 1 if $j=0$ and 2 if $j \geq 1$. Examples of eigenvectors of $-\Delta$ on a unit disk with Dirichlet boundary conditions are given in Figure 1.

To compute the eigenvectors and eigenvalues of $H=$ $-\Delta+\varepsilon V_{0}$, we use formulas (111) and (112). In this case the corresponding functionals are mock functionals as (111) and (112) do not take into account the degeneracy of the problem which means that the eigenvalues are of multiplicity two. The correct formulae require some complicated normalization constants given by (78).

In our case, we use the potentials

$$
\begin{aligned}
& V_{0}(r)=\frac{1}{r^{2} \chi_{\{r \leq \delta\}}}, \\
& V_{0}(r)=r \chi_{\{r \leq \delta\}},
\end{aligned}
$$

where $\delta<1$. 
TABLE 2: Value of $J_{N}^{V}$ on unit disk with $V=1 / r^{2}$.

\begin{tabular}{lccccc}
\hline$\varepsilon / \delta$ & 0.1. & 0.2. & 0.3. & 0.4 & 0.475. \\
\hline 0.01 & 0.499999996 & 0.499999997 & 0.499999763 & 0.499995606 & 0.499999756 \\
0.05 & 0.5 & 0.499999988 & 0.499998816 & 0.499987946 & 0.499998898 \\
0.1 & 0.5 & 0.499999975 & 0.499997638 & 0.499999650 & 0.499998085 \\
0.5 & 0.5 & 0.499999988 & 0.499998824 & 0.499988114 & 0.499998896 \\
1 & 0.499999999 & 0.499999997 & 0.499999764 & 0.499995642 & 0.499999756 \\
\hline
\end{tabular}

TABLE 3: Value of $J_{N}^{V}$ on unit disk with $V=r$.

\begin{tabular}{lllccc}
\hline$\varepsilon / \delta$ & 0.1. & 0.2. & 0.3. & 0.4 & 0.475. \\
\hline 0.01 & 0.5 & 0.5 & 0.499999995 & 0.499999759 & 0.499998584 \\
0.05 & 0.5 & 0.5 & 0.499999975 & 0.499998825 & 0.499999896 \\
0.1 & 0.5 & 0.5 & 0.499999950 & 0.499997720 & 0.499999794 \\
0.5 & 0.499999999 & 0.499999999 & 0.499999748 & 0.499991449 & 0.499999063 \\
1 & 0.499999997 & 0.499999998 & 0.499999496 & 0.499990010 & 0.499998365 \\
\hline
\end{tabular}

There are several important equalities to note here. For radial subsets $\omega$ of the form $\omega=\{(r, \theta) \in[0,1] \times[0,2 \pi] \mid \theta \in$ $\left.\omega_{0}\right\}$,

$$
\begin{aligned}
\int_{\omega} \phi_{j k m 0}(x)^{2} d x & =\int_{0}^{1} R_{j k}(r)^{2} r d r \int_{\omega_{0}} Y_{j m}(\theta)^{2} d \theta \\
& =\int_{\omega_{0}} Y_{j m}(\theta)^{2} d \theta
\end{aligned}
$$

since $\int_{0}^{1} R_{j k}(r)^{2} r d r=1$.

Matlab was used for computations, and Chebfun was utilized for the numerical computation of bessel functions and its derivatives. The integration of functions with explicit formulas was performed using the integral function in Matlab. The integration involving bessel functions was performed using the besselj function in Matlab. For the integration of the eigenvectors of $H=-\Delta+\varepsilon V_{0}$, the $2 \mathrm{D}$ trapezoid rule was used. We used a mesh size of 301 equal increments. Twentyfive eigenfunctions were computed.

Next, we consider the problem of maximizing the functional $J_{N}^{V}\left(\chi_{\omega}\right)$,

$$
J_{N}^{V}\left(\chi_{\omega}\right)=\inf _{1 \leq j \leq N} \int_{\omega} \phi_{j}^{2}(x) d x
$$

over all subsets satisfying $|\omega|=L|\Omega|$, for some $L \in(0,1)$; the argument of the maximum of which is called the optimal set. According to Propositions 3.9 and 4.5 of [6], for the $-\Delta$ operator, the maximum value of $J\left(\chi_{\omega}\right)=\inf _{1 \leq j \leq N} \int_{\omega} \phi_{j 0}^{2}(x)$ for radial subsets $\omega$ of the form $\omega=\{(r, \theta) \in[0,1] \times[0,2 \pi] \mid$ $\left.\theta \in \omega_{0}\right\}$ and measure $L \pi$ is $L$. In the case when $L=0.5$, the supremum is reached for all subsets $\omega$ of the form $\omega=$ $\left\{(r, \theta) \in[0,1] \times[0,2 \pi] \mid \theta \in \omega_{0}\right\}$ of measure $\pi / 2$, where $\omega_{\theta}$ is any measurable subset of $[0,2 \pi]$ such that $\omega$ and its symmetric image are complementary in $[0,2 \pi]$. To gain a better understanding of the case of the $H=-\Delta+\varepsilon V_{0}$ operator, we used $L=0.5$ and tested radial subsets of measure $0.5 \pi$ (or half the area of the total disk) satisfying Proposition 4.5 of [6].
In particular, we note results for $\omega_{0}=\{[0, \pi / 4] \cup[\pi / 2,3 \pi / 4] \cup$ $[\pi, 5 \pi / 4] \cup[3 \pi / 2,7 \pi / 4]\}$ and $N=25$.

The results are shown in Tables 2 and 3 ; the $\delta$ and $\varepsilon$ variables were varied. The values in the table show that, in all cases, the value of $J_{N}^{V}\left(\chi_{\omega}\right)$ is very close to 0.5 , which is the answer for the $-\Delta$ operator.

\section{Appendix}

\section{Convergence of Algorithms}

In order to provide an accurate numerical scheme, we also prove several theorems about $J^{V}\left(\chi_{\omega}\right)$ and the problem of maximizing the functional. First, we prove convergence of the truncated version of $J^{V}\left(\chi_{\omega}\right)$ for generic potentials.

Theorem A.1. Let

$$
\begin{aligned}
J_{N}^{V}\left(\chi_{\omega}\right) & =\inf _{1 \leq j \leq N} \int_{\omega} \phi_{j}^{2}(x) d x, \\
J_{N}^{V}(a) & =\inf _{1 \leq j \leq N} \int_{\Omega} a(x) \phi_{j}^{2}(x) d x
\end{aligned}
$$

Then, the following statements hold:

(1) For every measurable subset $\omega$ of $\Omega$, the sequence $\left(J_{N}^{V}\left(\chi_{\omega}\right)\right)_{N \in \mathbb{N}^{*}}$ is nonincreasing and converges to $J^{V}\left(\chi_{\omega}\right)$.

(2) The following equality holds:

$$
\lim _{N \rightarrow \infty} \max _{a \in \overline{\mathscr{M}}_{L}} J_{N}^{V}(a)=\max _{a \in \overline{\mathscr{M}}_{L}} J^{V}(a) .
$$

Moreover, whenever $\left(a^{N}\right)_{n \in \mathbb{N}^{*}}$ is a sequence of maximizers of $J_{N}^{V}$ in $\bar{M}_{L}$, then, up to a subsequence, this converges to a maximizer of $J$ in $\bar{M}_{L}$ for the weak star topology of $L^{\infty}$.

(3) Assume that $\Omega$ is a bounded analytic domain with boundary $\partial \Omega$. We have that, $\forall N \in \mathbb{N}^{*}$, the problem 
$\max _{\chi_{\omega} \in \bar{M}_{L}} J_{N}^{V}\left(\chi_{\omega}\right)$ has a unique solution $\chi_{\omega^{N}}$, where $\omega^{N} \in \mathscr{M}_{L}$. Moreover, the set $\omega^{N}$ is semianalytic and has a finite number of connected components.

We show that this relaxed problem allows for the determination of the observability constant under some assumptions on the flow.

Theorem A.2. Assume that there exists a subsequence of the sequence of probability measures $\mu_{j}=\phi_{j}^{2} d x$, which converges vaguely to the measure $(1 /|\Omega|) d x$ (Weak Quantum Ergodicity assumption with a potential). Then, the sequence of eigenfunctions $\phi_{j}$ is uniformly bounded in $L^{\infty}(\Omega)$ and

$$
\begin{aligned}
J^{V}\left(\chi_{\omega}\right) & =\sup _{\omega \in \mathscr{M}_{L}} \inf _{j \in \mathbb{N}^{*}} \int_{\omega} \phi_{j}^{2}(x) d x \\
& =\sup _{a \in \overline{\mathscr{M}}_{L}} \inf _{j \in \mathbb{N}^{*}} \int_{\Omega} a(x) \phi_{j}(x)^{2} d x=L .
\end{aligned}
$$

The assumptions of the above theorem are sufficient but not necessary to derive such a no-gap statement between the original formulation of the problem and the relaxed formulation. It is known that when $\Omega$ is a two-dimensional disk and $V(x) \equiv 0$, the same statement holds true, even though WQUE (weak quantum unique ergodicity) is not satisfied [6].

Proof of Theorem A.1. To formulate the proof, we use the same steps as in [6] in the proof of Theorem 4.9. These steps follow identically using the eigenfunctions $\phi_{j}$ of the $-\Delta+V$ operator instead of the eigenfunctions of the $-\Delta$ operator. We omit the steps here.

Proof of Theorem A.2. To conclude the proof, we use the same steps as in [6] in the proof of Theorem 3.5. These steps follow identically using the eigenfunctions $\phi_{j}$ of the $-\Delta+V$ operator instead of the eigenfunctions of the $-\Delta$ operator. We omit the steps here.

Lemma A.3. The convexified problem $\sup _{a \in \overline{\mathscr{M}}_{L}} J(a)$ has at least one solution and

$$
\sup _{a \in \overline{\mathscr{M}}_{L}} \inf _{j \in \mathbb{N}^{*}} \int_{\Omega} a(x) \phi_{j}^{2}(x) d x=L .
$$

The supremum is reached for the constant function $a(\cdot)=L$ on $\Omega$.

Proof of Lemma A.3. The first statement follows from the fact that $J(a)$ is upper semicontinuous for the $L^{\infty}$ topology. In order to prove the second statement, we use the Cesàro means of eigenfucntions. The constant function shows the fact that (A.4) is bounded below by $L$. We have

$$
\begin{aligned}
& \sup _{a \in \overline{\mathscr{M}}_{L}} \inf _{j \in \mathbb{N}^{*}} \int_{\Omega} a(x) \phi_{j}^{2}(x) d x \\
& \quad \leq \inf _{j \in \mathbb{N}^{*}} \frac{1}{N} \sum_{j=1}^{N} \int_{\Omega} a^{\star}(x) \phi_{j}^{2}(x) d x,
\end{aligned}
$$

where $a^{\star}$ is a solution of the convexified problem. By using a similar argument to that in the proof of Lemma 3.3 in [6] regarding the uniform $|\Omega|^{-1}$ limit of the sequence $N^{-1} \sum_{j=1}^{N} \phi_{j}^{2}$ of Cesàro means, one can show that (A.4) is bounded above by $L$ (the properties of Cesáro means for the eigenfunctions are trivially satisfied by the spectral theorem).

\section{Data Availability}

No data were used to support this study.

\section{Conflicts of Interest}

The authors declare that they have no conflicts of interest.

\section{Acknowledgments}

Alden Waters acknowledges support by EPSRC grant EP/L01937X/1.

\section{References}

[1] S. Kumar and J. H. Seinfeld, "Optimal location of measurements for distributed parameter estimation," IEEE Transactions on Automatic Control, vol. 23, no. 4, pp. 690-698, 1978.

[2] K. Morris, "Linear-quadratic optimal actuator location," Institute of Electrical and Electronics Engineers Transactions on Automatic Control, vol. 56, no. 1, pp. 113-124, 2011.

[3] O. Sigmund and J. S. Jensen, "Systematic design of phononic band-gap materials and structures by topology optimization," The Royal Society of London. Philosophical Transactions. Series A. Mathematical, Physical and Engineering Sciences, vol. 361, no. 1806, pp. 1001-1019, 2003.

[4] D. Ucinski and M. Patan, "Sensor network design for the estimation of spatially distributed processes," International Journal of Applied Mathematics and Computer Science, vol. 20, no. 3, pp. 459-481, 2010.

[5] M. van de Wal and B. de Jager, "A review of methods for input/output selection," Automatica, vol. 37, no. 4, pp. 487-510, 2001.

[6] Y. Privat, E. Trelat, and E. Zuazua, "Optimal observability of the multi-dimensional wave and Schrodinger equations in quantum ergodic domains," Journal of the European Mathematical Society, vol. 18, no. 5, pp. 1043-1111, 2016.

[7] F. Macia and G. Riviere, "Concentration and non-concentration for the schrodinger evolution on zoll manifolds," Communications in Mathematical Physics, vol. 345, no. 3, pp. 1019-1054, 2016.

[8] F. Macia, "Observability and quantum limits for the Schrödinger equation on the sphere," 2017, https://arxiv.org/ abs/1702.02066.

[9] F. Macia, "The Schrodinger flow on a compact manifold: Highfrequency dynamics, and dispersion," in Modern Aspects of the Theory of Partial Differential Equations, vol. 216 of Oper. Theory Adv. Appl., pp. 275-289, 2011.

[10] C. Laurent, "Global controllability and stabilization for the nonlinear Schrödinger equation on an interval," ESAIM-COCV, vol. 16, no. 2, pp. 356-379, 2010. 
[11] C. Laurent, "Global controllability and stabilization for the nonlinear Schrödinger equation on some compact manifolds of dimension 3," SIAM Journal on Mathematical Analysis, vol. 42, no. 2, pp. 785-832, 2010.

[12] L. Rosier and B.-Y. Zhang, "Local exact controllability and stabilizability of the nonlinear Schrodinger equation on a bounded interval," SIAM Journal on Control and Optimization, vol. 48, no. 2, pp. 972-992, 2009.

[13] L. Rosier and B.-Y. Zhang, "Exact boundary controllability of the nonlinear Schrödinger equation," Journal of Differential Equations, vol. 246, no. 10, pp. 4129-4153, 2009.

[14] Y. Privat, E. Trelat, and E. Zuazua, "Optimal observation of the one-dimensional wave equation," Journal of Fourier Analysis and Applications, vol. 19, no. 3, pp. 514-544, 2013.

[15] G. Lebeau, "Controle de l'equation de Schrodinger," Journal de Mathématiques Pures et Appliquées, vol. 71, no. 3, pp. 267-291, 1992.

[16] S. Jaffard, "Control interne exact des vibrations d'une plaque rectangulaire Portugal," Portugaliae Mathematica, vol. 47, no. 4, pp. 423-429, 1990.

[17] A. Haraux, "Series lacunaires et control semi-interne des vibrations d'une plaque rectangulaire," Journal de Mathématiques Pures et Appliquées, vol. 68, no. 4, pp. 457-465, 1989.

[18] V. Komornik, "On the exact internal controllability of a Petrowsky system," Journal de Mathématiques Pures et Appliquées, vol. 71, no. 4, pp. 331-342, 1992.

[19] N. Burq and M. Zworski, "Geometric control in the presence of a black box," Journal of the American Mathematical Society, vol. 17, no. 2, pp. 443-471, 2004.

[20] N. Burq and M. Zworski, "Control for Schrodinger operators on tori," Mathematical Research Letters, vol. 19, no. 2, pp. 309-324, 2012.

[21] N. Anantharaman and F. Macia, "Semiclassical measures for the Schrodinger equation on the torus," Journal of the European Mathematical Society, vol. 16, no. 6, pp. 1253-1288, 2014.

[22] N. Anantharaman, C. Fermanian-Kammerer, and F. Macia, "Semiclassical completely integrable systems: long-time dynamics and observability via two-microlocal Wigner measures," American Journal of Mathematics, vol. 137, no. 3, pp. 577-638, 2015.

[23] L. Jin, "Control for Schrodinger equation on Hyperbolic surfaces," https://arxiv.org/pdf/1707.04990.pdf.

[24] N. Anantharaman, M. Leautaud, and F. Macia, "Wigner measures and observability for the Schrodinger equation on the disk," Inventiones Mathematicae, vol. 206, no. 2, pp. 485-599, 2016.

[25] H. Gimperlein and A. Waters, "A deterministic optimal design problem for the heat equation," SIAM Journal on Control and Optimization, vol. 55, no. 1, pp. 51-69, 2017.

[26] J. Ilmavirta and A. Waters, "Recovery of coefficients for the acoustic wave equation from phaseless measurements," Communications in Mathematical Sciences, 2018.

[27] A. Strohmaier, "Computation of eigenvalues, spectral zeta functions and zeta-determinants on hyperbolic surfaces," in Geometric and computational spectral theory, vol. 700 of Contemp. Math., pp. 177-205, Amer. Math. Soc., Providence, RI, 2017.

[28] E. B. Davies, Spectral Theory and Differential Operators, vol. 42, Cambridge University Press, Cambridge, UK, 1989.

[29] T. Kato, Perturbation Theory, Springer-Verlag Heidelberg, Berlin, Germany, 1980.
[30] W. M. Greenlee, "Singular perturbation of simple eigenvalues," Rocky Mountain Journal of Mathematics, vol. 6, no. 4, pp. 575584, 1976.

[31] V. Guillemin, E. Legendre, and R. Sena-Dias, "Simple spectrum and Rayleigh quotients," in Geometric and spectral analysis, P. Albin, D. Jakobson, and F. Rochon, Eds., vol. 630 of Contemp. Math., pp. 33-44, Amer. Math. Soc., Providence, RI, 2014.

[32] K. Uhlenbeck, "Generic properties of eigenfunctions," American Journal of Mathematics, vol. 98, no. 4, pp. 1059-1078, 1976.

[33] D. D. Bleecker and L. C. Wilson, "Splitting the spectrum of a Riemannian manifold," SIAM Journal on Mathematical Analysis, vol. 11, no. 5, pp. 813-818, 1980.

[34] J. Conway, A Course in Functional Analysis, Graduate Texts in Mathematics, Springer, New York, NY, USA, 2nd edition, 1990. 


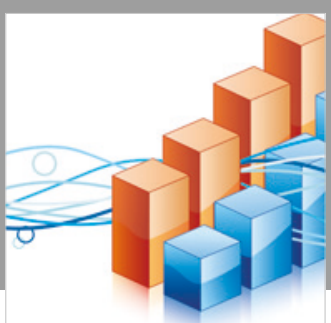

Advances in

Operations Research

\section{-n-m}
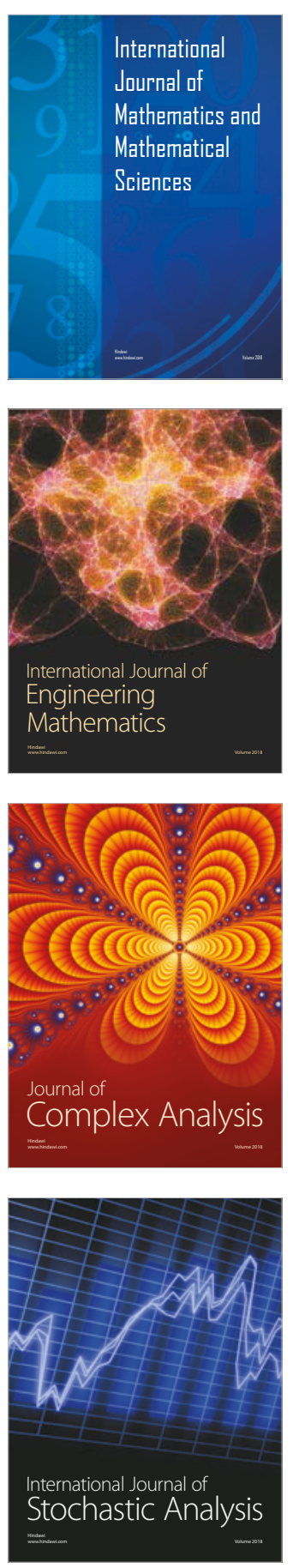
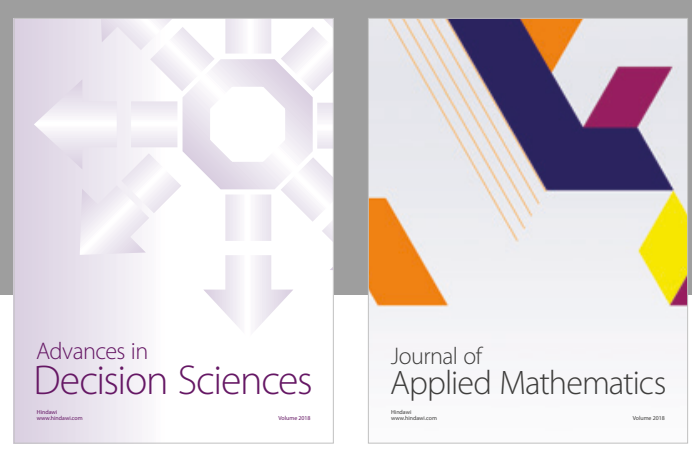

Journal of

Applied Mathematics
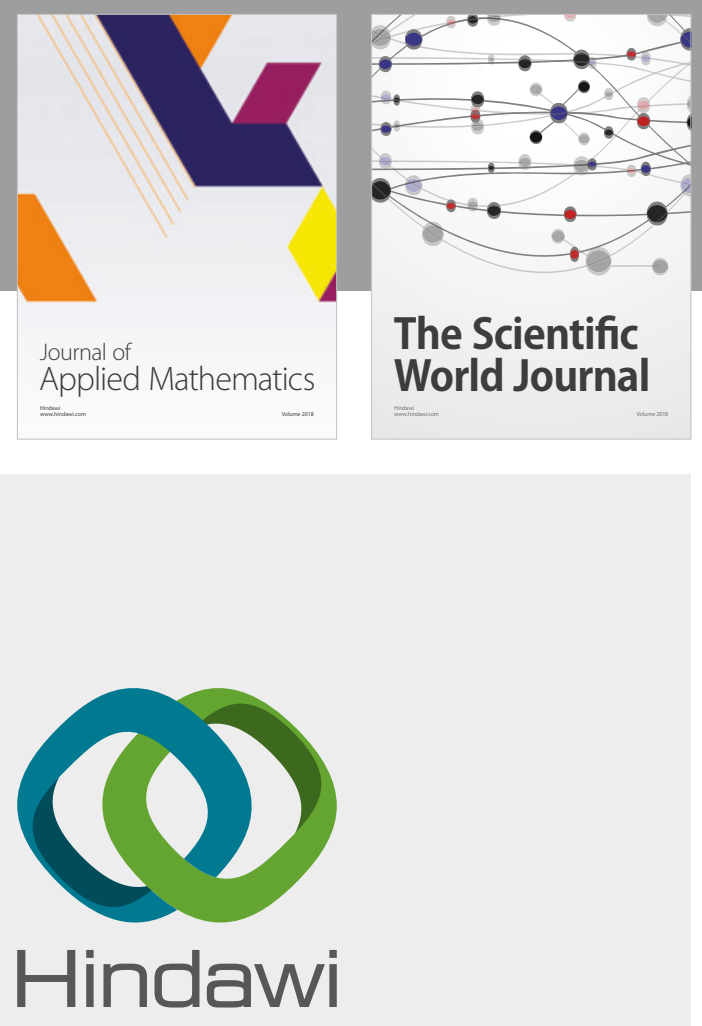

Submit your manuscripts at

www.hindawi.com

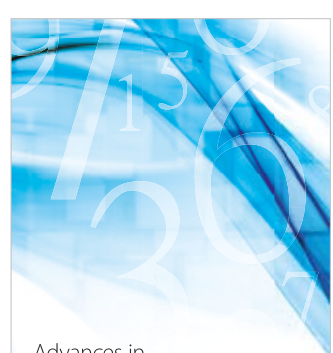

Advances in
Numerical Analysis
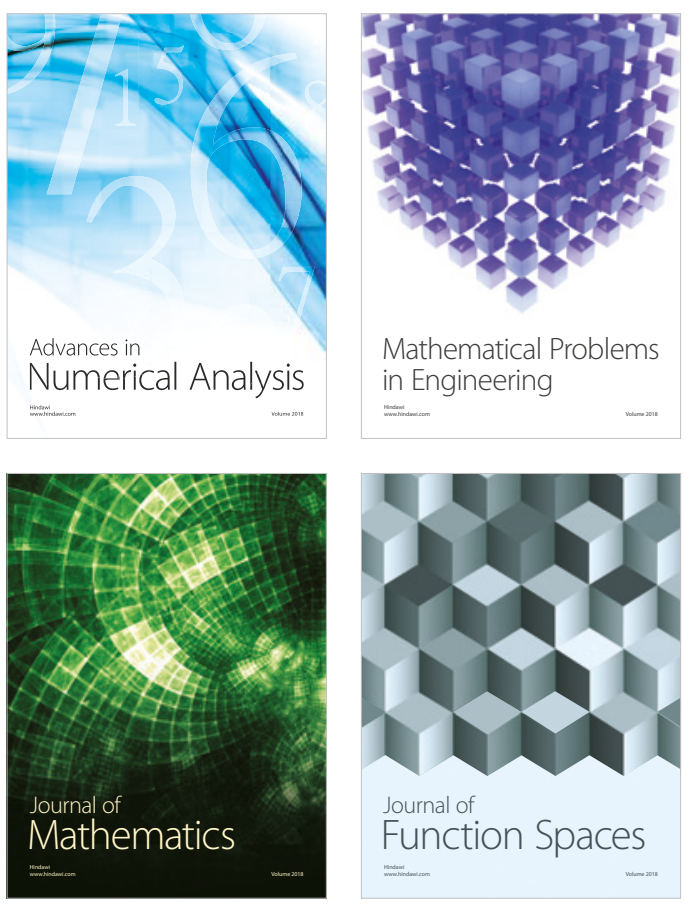

Mathematical Problems in Engineering

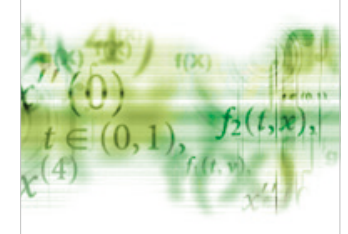

International Journal of

Differential Equations

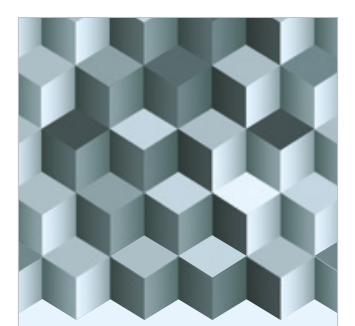

Journal of

Function Spaces
The Scientific

World Journal

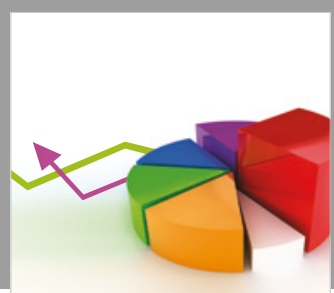

Journal of

Probability and Statistics
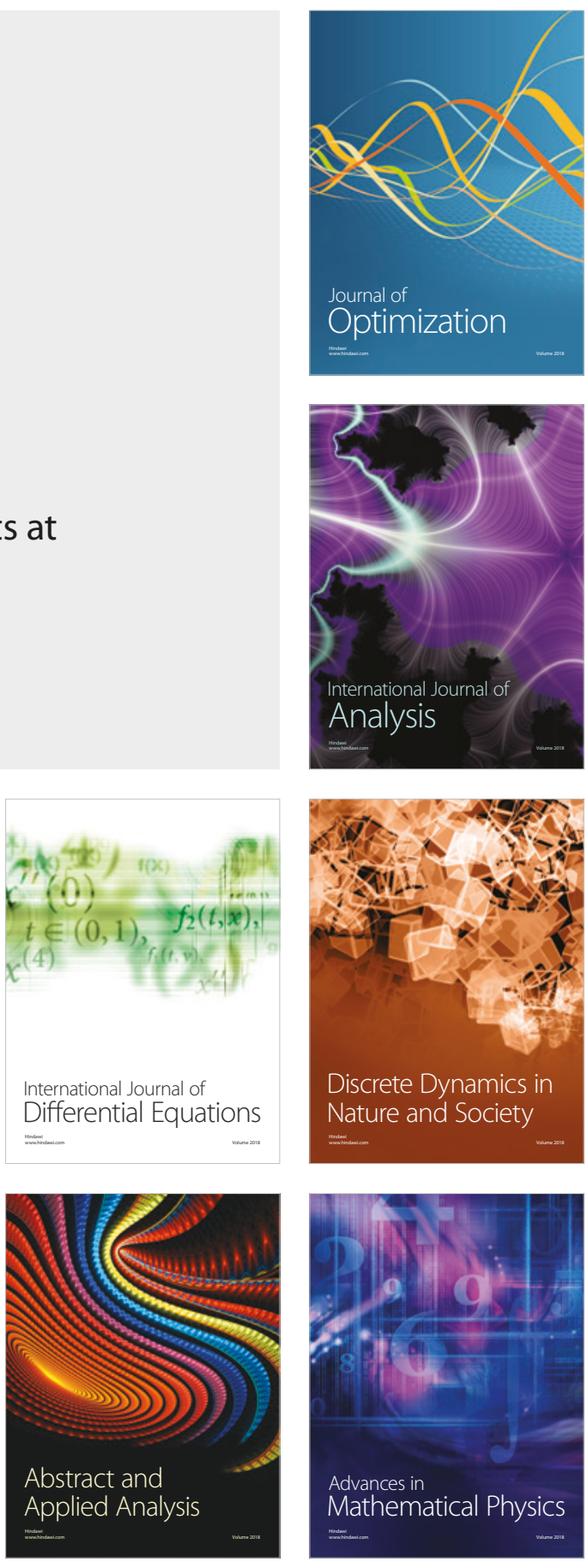